\title{
Molecular basis for gating and regulation of cardiac ryanodine receptor: underlying mechanisms for gain- and loss-of function mutations
}

Takuya Kobayashi ${ }^{1,5}$, Akihisa Tsutsumi ${ }^{2,5}$, Nagomi Kurebayashi ${ }^{1}$, Kei Saito ${ }^{3}$, Masami Kodama ${ }^{4}$, Takashi Sakurai ${ }^{1}$, Masahide Kikkawa ${ }^{2}$, Takashi Murayama ${ }^{1, *}$ and Haruo Ogawa ${ }^{4,6, *}$

${ }^{1}$ Department of Cellular and Molecular Pharmacology, Juntendo University Graduate School of Medicine, Tokyo, Japan

${ }^{2}$ Department of Cell Biology and Anatomy, Graduate School of Medicine, The University of Tokyo, Tokyo, Japan

${ }^{3}$ Department of Life Sciences, Graduate School of Arts and Sciences, The University of Tokyo, Tokyo, Japan

${ }^{4}$ Institute for Quantitative Biosciences, The University of Tokyo, Tokyo, Japan

${ }^{5}$ These authors contributed equally

${ }^{6}$ Lead Contact

*Correspondence: Email: takashim@juntendo.ac.jp (T.M.); haru@iqb.u-tokyo.ac.jp (H.O.) 


\section{Summary}

Cardiac ryanodine receptor (RyR2) is a large $\mathrm{Ca}^{2+}$ release channel in the sarcoplasmic reticulum and indispensable for excitation-contraction coupling in the heart. RyR2 is activated by $\mathrm{Ca}^{2+}$ and RyR2 mutations have been implicated in severe arrhythmogenic heart diseases. Yet, the structural basis underlying channel opening and how mutations affect the channel remain unknown. Here, we combined high-resolution structures determined by cryo-electron microscopy with quantitative functional analysis of channels carrying various mutations in specific residues. We demonstrated two fundamental mechanisms for channel regulation: interactions close to the channel pore stabilize the channel to prevent hyperactivity and a series of interactions in the surrounding regions is necessary for channel opening upon $\mathrm{Ca}^{2+}$ binding. Mutations at the residues involved in the former and the latter mechanisms cause gain-of-function and loss-of-function, respectively. Our results reveal gating mechanisms of the RyR2 channel and alterations by pathogenic mutations at the atomic level.

\section{Keywords}

Ryanodine receptor, Membrane protein, calcium channel, cryo-electron microscopy, loss-offunction, gain-of-function, mutation, cardiac muscle, ER/SR membrane, excitation-contraction coupling 


\section{Introduction}

Cardiac muscle contraction is triggered by an influx of $\mathrm{Ca}^{2+}$ into the cytosol through L-type voltage-dependent $\mathrm{Ca}^{2+}$ channels in the plasma membrane. This increase in $\mathrm{Ca}^{2+}$ activates the cardiac ryanodine receptor (RyR2) in the sarcoplasmic reticulum to release large amount of $\mathrm{Ca}^{2+}$, a process known as $\mathrm{Ca}^{2+}$-induced $\mathrm{Ca}^{2+}$ release (CICR) (Endo, 2009; Fabiato and Fabiato, 1978). RyR2 is a homotetramer and the largest ion channel identified to date (Bers, 2004; Santulli et al., 2018). Its total molecular weight is $\sim 2.2 \mathrm{Mda}$, with each monomer composed of $\sim 5,000$ amino acid residues and comprising a large $\mathrm{N}$-terminal mushroom-like structure of the cytoplasmic domain and $\mathrm{C}$-terminal transmembrane (TM) region (Clarke and Hendrickson, 2016; Zalk and Marks, 2017).

In human RyR2, nearly 300 pathogenic mutations have been reported as arrhythmogenic heart diseases, including catecholaminergic polymorphic ventricular tachycardia (CPVT) (Kawamura et al., 2013; Pérez-Riera et al., 2018; Priori et al., 2002; Tester et al., 2004), left ventricular non compaction (LVNF) (Nozaki et al., 2020; Ohno et al., 2014; Tang et al., 2012), and idiopathic ventricular fibrillation (IVF) (Fujii et al., 2017; Jiang et al., 2007; Paech et al., 2014). These mutations alter channel activity to cause either gain-of-function or loss-of-function (Benkusky et al., 2004; Priori and Chen, 2011). However, it remains unclear how these mutations affect the channels at the molecular level.

The first reported structure of RyR2, derived from pig heart, was in the closed state in the presence of EGTA and in the open state with $\mathrm{Ca}^{2+}$ and PCB95 (Peng et al., 2016). This was followed by reports on RyR2 activity modulation by regulatory proteins, such as calmodulin (Gong et al., 2019) and FKBP12.6 (Chi et al., 2019). These reports along with prior studies on RyR1 (see (Ogawa et al., 2020) for a recent review) provided insights into RyR2 domain organizations, conformational changes upon channel opening, and the binding sites for ligands and regulatory proteins. Structural analysis has indicated the importance of intra/inter-domain interactions for long-range allosteric regulation of channel gating, yet the roles of individual interaction remains unknown. Thus, the fundamental mechanism underlying channel opening at the atomic level remains to be elucidated. Moreover, it remains unclear how pathogenic mutations alter the channel activity.

To identify key movements and interactions in the channel opening, we here obtained highresolution structures of recombinant mouse RyR2 by cryo-electron microscopy (EM) single-particle analysis. Then, functional analysis was conducted with RyR2 channels carrying mutations in aminoacid residues in the proposed interactions to clarify their roles during channel gating. Moreover, structures of two mutant channels were determined to address molecular mechanism of alteration. We demonstrated two fundamental mechanisms for channel regulation of RyR2. Interactions for stabilization of the channel in the closed state are located close to the channel pore, while a series of 
interactions located in the surrounding regions is necessary for channel opening upon $\mathrm{Ca}^{2+}$ binding. Disruption of the former and the latter interactions causes loss-of-function and gain-of-function, respectively. Our results reveal mechanisms underlying channel opening upon $\mathrm{Ca}^{2+}$ binding and explain how pathogenic mutations alter channel activity at the atomic level.

\section{RESULTS}

\section{Overall conformational changes in RyR2 associated with $\mathrm{Ca}^{2+}$ binding}

Recombinant mouse RyR2, expressed and purified from HEK293 cells using FKBP12.6 affinity chromatography (Cabra et al., 2016) formed homogeneous tetrameric channels (Figures. S1A and S1B). To precisely trace the $\mathrm{Ca}^{2+}$-induced RyR2 structural changes, a fully open state without any activating ligands other than $\mathrm{Ca}^{2+}$ is necessary. Thus, we adopted high salt conditions, which markedly enhance channel activity with $\mathrm{Ca}^{2+}$ alone (Figures S1C-S1E) (Meissner, 1994; Ogawa, 1994). We performed cryo-EM single-particle analysis in the presence of $1 \mathrm{mM}$ EGTA and $100 \mu \mathrm{M}$ $\mathrm{Ca}^{2+}$ for closed and open states, respectively. Three-dimensional (3D) classification including focused classification analysis using the TM region revealed two and three classes in the presence of EGTA and $\mathrm{Ca}^{2+}$, respectively (Figures S1F and S1G; Table S1). No major differences were observed among the classes both in the closed and open states. Therefore, subsequent analysis was performed using the class with the highest resolution (before the final classification in the closed state and class 1 in the open state; Table S1). The overall resolution of the final model was $3.3 \AA$ and $3.45 \AA$ for the closed and open states, respectively, and the local resolution for the TM region in the closed state was better than $2.9 \AA$ (Figures S1F and S1G), which allowed us to build precise atomic models and identify specific residues important for channel gating (Figures S1H and S1I). In fact, it was confirmed that the pore was open only by addition of $100 \mu \mathrm{M}$ of $\mathrm{Ca}^{2+}$ (Figure S2A). Conformational changes between closed and open states were large and spanned the entire molecule (Figures 1A, 1B, S2BS2D; Video S1). Changes associated with $\mathrm{Ca}^{2+}$ binding were analyzed in three layers with different heights parallel to the membrane, i.e., the C-terminal domain (CTD), U-motif, and S4-S5 layers (Figures 1C-1E; Video S1). Upon $\mathrm{Ca}^{2+}$ binding, CTD rotated clockwise (viewed from cytoplasmic side) (Figure 1C), U-motif rotated clockwise toward the S2-S3 domain (Figure 1D), and S6 leaned outward from the center to open the gate accompanied by rearrangements of S1-S4 helices and outward movement of the S4-S5 linker (Figure 1E). The C-terminal side of the central domain (Ccentral), U-motif, CTD, and cytoplasmic S6 (S6 $6_{\text {cyto }}$ ) were tightly attached to each other in both closed and open states (Figures $1 \mathrm{~F}, \mathrm{~S} 2 \mathrm{E}$, and S2F; Video S2) and rotated together $9.8^{\circ}$ clockwise with respect to the axis approximately parallel to $\mathrm{S} 6$ (Figure $1 \mathrm{G}$ ). In contrast, the $\mathrm{N}$-terminal side of the 
central domain ( $\mathrm{N}$-central) did not follow the rotation due to splitting the central domain into two parts with $\mathrm{G} 3987$ as the pivot (Figure 1F). Since the rotation axis was close to the $\mathrm{Ca}^{2+}$-binding site, slight movements caused by $\mathrm{Ca}^{2+}$ binding resulted in very large movements around $\mathrm{S} 6$ (Figure 1G).

Given these series of movements, we hypothesized the gating mechanism of RyR2 as follows: (1) the rotation of the C-central/U-motif/S6 $6_{\text {cyto }} / \mathrm{CTD}$ complex upon $\mathrm{Ca}^{2+}$ binding leads to two downstream pathways for channel pore opening; (2) the $S 6_{\text {cyto }}$ movement leads to the outward leaning of S6; (3) the U-motif movement leads to sequential movements of the S2-S3 domain and TM segments, causing outward movement of the S4-S5 linker and creating a space where S6 can lean into; and (4) the channel pore finally opens by combining these two independent pathways (Figure $1 \mathrm{H}$ ). To prove this hypothesis, we performed detailed structural analysis and functional studies.

\section{U-motif/S2-S3 domain interaction is key for signal transduction in response to $\mathrm{Ca}^{2+}$ binding}

In our hypothesis, the U-motif/S2-S3 domain interaction is important in transducing the rotation of the U-motif in response to TM segment movement (Figure 1D; Videos S1 and S2). We found three key interactions between the U-motif and S2-S3 domain formed by hydrogen bonds or salt bridges (Figures 2A-2C and S3A; Video S3). E4198-K4593-S4167 was evident in the open state, whereas Y4498-K4593 was only formed in the closed state, indicating that K4593 switches the interacting partner between the two states. Additionally, E4193-R4607 was stable in both states.

To clarify the roles of hydrogen bonds/salt bridges in channel gating, functional assays were conducted with recombinant RyR2 carrying mutations at the specific residues (Table 1). $\mathrm{Ca}^{2+}{ }_{-}$ dependent $\left[{ }^{3} \mathrm{H}\right]$ ryanodine binding was used in functional analysis as it reflects channel activity (Fujii et al., 2017; Murayama et al., 2018b). Wild-type (WT) RyR2 exhibited biphasic $\mathrm{Ca}^{2+}$-dependent $\left[{ }^{3} \mathrm{H}\right]$ ryanodine binding, whereas alanine substitution at K4593 markedly reduced the binding (Figure 2D). Two pathogenic mutations, K4593Q and K4593R, also led to loss of binding (Figures 2E and S3B). Similarly, binding was severely reduced after alanine substitutions (Y4498A, S4167A, and $\mathrm{E} 4198 \mathrm{~A}$ ) and pathogenic mutation (S4167P) in interacting partners (Figures 2D, 2E and S3B). The behaviors of mutant RyR2 channels were also evaluated in live cells by monitoring cytoplasmic and endoplasmic reticulum (ER) luminal $\mathrm{Ca}^{2+}$ homeostasis (Fujii et al., 2017; Uehara et al., 2017). In WT RyR2-expressing HEK293 cells, spontaneous $\mathrm{Ca}^{2+}$ oscillations occurred with a concomitant decrease in $\left[\mathrm{Ca}^{2+}\right]_{E R}$, indicating $\mathrm{Ca}^{2+}$ release from the ER via RyR2 channels (Figure 2F, left). In contrast, RyR2 channels carrying the K4593A mutation showed no such $\mathrm{Ca}^{2+}$ oscillations with increased $\left[\mathrm{Ca}^{2+}\right]_{E R}$, indicating a loss-of-function of the channel (Figure 2F, right). Similar results were obtained with other 
substitutions in S4167, E4198, and K4593 (Figure 2G). Altogether, these results suggest that both E4198-K4593-S4167 and Y4498-K4593 interactions are important for channel opening. We also evaluated the E4193-R4607 interaction and found that alanine substitutions (E4193A and R4607A) and pathogenic mutations (R4607Q and R4607W) led to a loss-of-function of the channel (Figures 2D-2G, S3B). These residues and surrounding sequences are well conserved in all three mammalian RyR subtypes (Figure 2H). These findings indicate that three interactions above $\mathrm{S} 2$ in the U-motif/S2S3 domain interface play a critical role in transducing the $\mathrm{Ca}^{2+}$-binding signal to $\mathrm{S} 2$, and that loss of these interactions results in a loss of channel function.

\section{Movements of the S1-S4 bundle lead to outward movement of the S4-S5 linker}

Movement of the S2-S3 domain appears to cause S2 movement (see Figure $2 \mathrm{C}$ and video S3). This leads to the coordinated $7.6^{\circ}$ clockwise rotation of S1, S3, and S4 (Figures 1E, 3A-3C, S4AS4C; Videos S1 and S4). S1, S2, S3, and S4 are arranged in a circle, placed at equal intervals in a clockwise direction (Figures 3A-3C, S4A-S4C). We found interactions between S1 and S2 (S1/S2), $\mathrm{S} 2 / \mathrm{S} 3, \mathrm{~S} 3 / \mathrm{S} 4$, and S1/S4, all of which were maintained in both closed and open states (Figures $3 \mathrm{~A}$ and 3B, S4A-S4C; Video S4). The hydrophobic interactions between F4497 and L4592 at S1/S2; hydrogen bond between Y4589 and D4715 at S2/S3; hydrogen bond between Y4720 and D4744 at S3/S4; and the salt bridge between R4501 and D4744 at S1/S4 appear to bundle the four TM segments into one (i.e., S1-S4 bundle).

Alanine substitution of residues involved in S1/S2 (F4497 and L4592) and S2/S3 (Y4589 and D4715) interactions exhibited significantly reduced $\left[{ }^{3} \mathrm{H}\right]$ ryanodine binding (Figures 3D, 3E, and S4G), loss of $\mathrm{Ca}^{2+}$ oscillations, and increased $\mathrm{ER} \mathrm{Ca}^{2+}$ (Figures $3 \mathrm{~F}$ and $3 \mathrm{G}$ )-all indicative of loss-of-function. The pathogenic mutant F4497C also resulted in loss-of-function (Figures 3E, 3G and S4G). Thus, S1/S2 and S2/S3 interactions are necessary for channel opening. In contrast, alanine substitution or pathogenic mutations of residues involved in S1/S4 (R4501 and D4744) and S3/S4 (Y4720 and D4744) interactions caused gain-of-function of the channel with increased $\left[{ }^{3} \mathrm{H}\right]$ ryanodine binding and reduction in ER Ca ${ }^{2+}$ (Figures 3D-3G, and S4G). Especially, D4744A, which is involved in both interactions, exhibited greatly increased $\left[{ }^{3} \mathrm{H}\right]$ ryanodine binding with an enhanced $\mathrm{Ca}^{2+}$ sensitivity for activation and loss of $\mathrm{Ca}^{2+}$ inactivation (Figures 4D, S4I, and S4J). These residues are well conserved in all the mammalian RyR subtypes (Fig. 30).

Rotation of the S1-S4 bundle causes movement of the S4-S5 linker. We found that the upper part of $S 4$ rotated $40^{\circ}$ counterclockwise to form an a-helix upon channel opening, which dramatically changed the position of F4749 (Figures 3H-3J and S4A-S4F; Video S4). The S4-S5 linker, which is 
unfolded and significantly bent in the direction of S6 in the closed state, rewinds to an a-helix and moves outward (Figures S4D-S4F; Video S4). A hydrophobic interaction between F4749 in the upper part of S4 and L4505 in S1 was identified in the closed state (Figures 3H and S4A).

Alanine substitutions of residues involved in hydrophobic interactions (L4505A and F4749A) and the pathogenic mutant L4505P caused gain-of-function with increased $\mathrm{Ca}^{2+}$ sensitivity and loss of $\mathrm{Ca}^{2+}$ inactivation (Figures 3K-3N, S4H-S4J). Notably, no additive effects were observed upon binding of $\left[{ }^{3} \mathrm{H}\right]$ ryanodine to double mutant L4505A_F4749A (Figure S4H), supporting an interaction between the two residues. Whereas $L 4505$ is conserved in all the mammalian RyR subtypes, F4749 is mutated to valine in the RyR1 subtype (Figure 30). However, unlike alanine mutant, F4749V exhibited biphasic $\mathrm{Ca}^{2+}$ dependent $\left[{ }^{3} \mathrm{H}\right]$ ryanodine binding with slightly reduced peak value (Figures $\mathrm{S} 4 \mathrm{H}$ ).

Taking these findings into consideration, we hypothesized a channel opening mechanism in which a series of movements of S4-S5 linker regulate channel opening. In the absence of $\mathrm{Ca}^{2+}$, the channel is stabilized in the closed state as the S4-S5 linker is locked by the "stopper," in form of L4505-F4749 interaction, which prevents a-helix formation of the upper part of S4. The L4505-F4749 interaction is supported by $\mathrm{S} 1 / \mathrm{S} 3$ and $\mathrm{S} 3 / \mathrm{S} 4$ interactions that keep the two residues appropriately placed. Upon $\mathrm{Ca}^{2+}$ binding, clockwise rotation of the S1-S4 bundle induced by U-motif/S2-S3 domain interaction alters the relative positions of $\mathrm{L} 4505$ and F4749 to release the stopper. This allows $\alpha$-helix formation of the upper part of S4 and subsequent outward movement of the S4-S5 linker to open the channel. Loss of necessary interactions, such as that of the U-motif/S2-S3 domain (Figures $2 \mathrm{~A}-2 \mathrm{C}$ ), $\mathrm{S} 1 / \mathrm{S} 2$, and S2/S3 (Figures 3A-3C), cannot release the stopper, leading to loss-of-function (Figures 2D-2G and 3D-3G). Conversely, loss of interactions that support S4, such as S1/S4 and S3/S4 (Figures $3 \mathrm{~A}-3 \mathrm{C}$ and $3 \mathrm{H}-3 \mathrm{~J}$ ) destabilize the channel in the closed state and impart a gain-of-function of the channel (Figures 3D-3G and 3K-3N).

\section{U-motif/S6yto/CTD interaction plays key role in stabilizing the channel in the closed state}

The U-motif/S6 $6_{\text {cyto }} / C T D$ complex was stable in both the closed and open states (Figure 1F). We found a close contact between U-motif and $S 6_{\text {cyto }}$ as observed in the closed state structure of RyR1 (Yan et al., 2015) (Figures 4A, 4B, and S5A; Video S5). In the closed state, F4173, V4176, and N4177 in the U-motif and Q4875 and V4879 in S6cyto faced each other forming van der Waals interactions, and were surrounded by Q4876 and Q4878 from S6 (Figure 4B; Video S5). In the open state, S6 cyto $_{\text {c }}$

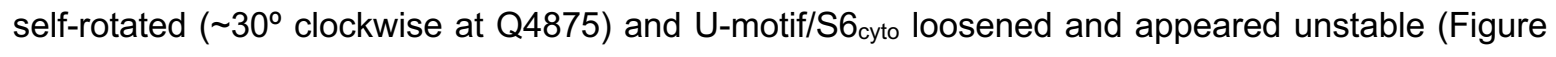
4C and S5B; Video S5). Buried surface area analysis calculated by CNS (Brünger et al., 1998) demonstrated that the U-motif/S6 $6_{\text {cyto }}$ interface in the open state $\left(362 \AA^{2}\right)$ was smaller than in the 
closed state $\left(514 \AA^{2}\right)$. The U-motif also interacted with CTD via F4888, where it penetrated partly into the hydrophobic pocket formed by U-motif F4171, 14172, and V4175 and CTD L4914 (Figure 4D and S5C; Video S5). The U-motif/CTD interaction was stable in both states.

All alanine-substituted mutants of residues interacting between U-motif and $\mathrm{S} 6_{\text {cyto }}$ as well as two CPVT mutations (N4177S and N4177Y) led to gain-of-function (Figures 4E-4H) with mild enhancement in $\mathrm{Ca}^{2+}$ sensitivity and severe loss of $\mathrm{Ca}^{2+}$ inactivation (Figures S5D, S5F and S5G). Alanine substitution and pathogenic mutation of F4888 exhibited severe gain-of-function with increased $\mathrm{Ca}^{2+}$ sensitivity and loss of $\mathrm{Ca}^{2+}$ inactivation (Figures 4I-4L, S5E-S5G). Alanine substitution of F4171, 14172, V4175, and L4914-involved in the hydrophobic pocket-also led to milder gain-offunction of the channel than F4888 mutants (Figures 4I-4L, S5E-S5G). Notably, no additive effects were observed with N4177A and F4888A, indicating that U-motif/S6 cyto and U-motif/CTD interactions are involved in the common pathways (Figure S5B). All the residues thought to be involved in the $U$ motif/S6cyto/CTD interactions and surrounding sequences are conserved in mammalian three RyR subtypes (Figure 4M). These findings suggest that interactions within the U-motif/S6 $6_{\text {cyto }} / C T D$ complex play a key role in stabilizing the closed state.

\section{Structural basis of the gain-of-function mutations}

To elucidate the structural changes associated with mutations, we conducted structural analysis of two gain-of-function mutants (F4888A and F4749A) (Figures S6 and S7). The density for phenyl group of each mutant disappeared clearly (Figures S6B, S6C, S7C, and S7D). Two different classes (closed/open-like structures) were obtained for both mutants in the presence of EGTA (Figures S6 and S7) and $\mathrm{Ca}^{2+}$ was bound in the open-like structures. This was not observed for the WT structure in the presence of EGTA, reflecting the result of increasing $\mathrm{Ca}^{2+}$ sensitivity in both mutants (Figures $3 \mathrm{~K}$ and $4 \mathrm{I})$. We also determined the structure in the presence of $100 \mu \mathrm{M} \mathrm{Ca}^{2+}$ for F4749A, and found essentially the same structure as that of open-like F4749A in the presence of EGTA. The overall resolution of the final models was 3.2-3.8 $\AA$ (Figures S6A, S7A, and S7B, and Table S1), which was sufficient to analyze structural changes induced by mutations (Figures S6B, S6C, S7C, and S7D).

In the closed structure of F4888A, appreciable change was detected in the U-motif (Figures $5 \mathrm{~A}$, $5 \mathrm{~B}$, and S6D; Video S6) with no differences in the TM segments and the S4-S5 linker (Figure S6E). Removal of the phenyl group created an extra space above F4171, causing the a-helix in the Nterminal U-motif to rotate $\sim 15^{\circ}$ clockwise around F4171 with respect to the WT in the closed state (Figures 5A and 5B; Video S6). Consequently, F4171 moved up to fill the space and the interaction between U-motif and $S 6_{\text {cyto }}$ became loose, as if the mutant partially mimicked the U-motif 
conformational change of the open state WT (Fig. 5A and 5B; Video S6). In the open structure, there was a parallel movement of the U-motif, S2-S3 domain, and S1-S4 toward the outside (Figures 5D and $5 \mathrm{E}$ ), which differed from the rotational motion observed in the WT structural change from closed to open states. The S4-S5 linker, S5, and S6 showed only a slight outward movement (Figures 5E, $\mathrm{S} 6 \mathrm{~F}$, and S6G). Movement of the U-motif in the mutant created a different environment from that of the open state WT U-motif/S6 $6_{\text {cyto }}$ interaction (Figure 5F). Moreover, the buried surface area of the Umotif/S6 cyto interface (335 $\left.\AA^{2}\right)$ was smaller than that of the open state WT (362 $\left.\AA^{2}\right)$. These findings are consistent with the idea that the movement of the U-motif is restricted by $S 6_{\text {cyto }}$ to stabilize the closed state.

In the closed structure of F4749A, removal of the phenyl group had no effect on the structure, and there was no appreciable structural difference from the WT in TM segments and the S4-S5 linker as well as overall structure (Figures 5C and S7E). Although we hypothesized that F4749 and L4505 form a stopper to prevent the rotation of upper part of S4 and subsequent movement of S4-S5 linker (Figure 3), no structural change was detected in the S4-S5 linker in the F4749A mutant. This evidence suggests that the destruction of the stopper itself does not induce spontaneous conformation changes in the S4-S5 linker. In the open state of F4749A, several structural changes were detected (Figure $\mathrm{S} 7 \mathrm{~F}$ ); subtle movements of the S4-S5 linker and S6 (Figures S7G and S7H), a parallel movement of the U-motif, S2-S3 domain (Figure S7I), S1-S4 toward the outside (Figures S7G-S7I), and altered the U-motif/S6 cyto interaction (Figure S7J). Thus, similar structural changes were observed for the two mutants with different points of action and this gain-of-function occurred via a common mechanism.

\section{Summary of the $\mathrm{Ca}^{2+}$ induced movements in the RyR2}

Here, we summarize the channel opening mechanism associated with $\mathrm{Ca}^{2+}$ binding of RyR2 determined by high-resolution cryo-EM structures: (1) binding of $\mathrm{Ca}^{2+}$ rotates the U-motif/S6 $6_{\text {cyto }} / \mathrm{CTD}$ complex by $9.8^{\circ}$ along with the axis which is $40 \AA$ apart from S6yto; (2) extruding the S2-S3 domain by the U-motif causes S2 to move; (3) movement of S2 rotates the S1-S4 bundle by $7.6^{\circ}$ to release the stopper comprising F4749/L4505; (4) rotation of the upper part of S4 allows outward movement of the S4-S5 linker, creating a space where S6 can lean into; and (5) rotation of S6 $6_{\text {cyto }}$ by $30^{\circ}$ causes the outward leaning of $S 6$ by $7.8 \AA$ (Figures $5 \mathrm{G}$ and $5 \mathrm{H}$ ). Thus, the sequential rotations of domains/ahelices and domain-domain interactions may occur in conformation changes associated with $\mathrm{Ca}^{2+}$ binding.

\section{Discussion}


By combining high-resolution cryo-EM structures and quantitative functional analysis of mutant channels, we successfully clarified the gating mechanism of RyR2 upon $\mathrm{Ca}^{2+}$ binding. Figure 6A shows a schematic diagram of the channel core domain of RyR2 with details of the important interactions identified in this study. Mutations of residues involved in the signaling pathway for outward movement of the S4-S5 linker caused loss-of-function, whereas those supporting the stopper (i.e., S1/S3/S4 and S1/S4 interactions) or the U-motif/CTD/S6 $6_{\text {cyto }}$ complex led to gain-of-function. Given the locations of these mutations, it becomes apparent that interactions close to the channel pore are important for stabilizing the channel in the closed state and those in the surrounding region are essential for channel opening.

The present results can reasonably explain the gating mechanism of RyR2 channel and how pathogenic or alanine mutations affect the channel activity (Figures 6B-6D). In the closed state of the WT channel, the S4-S5 linker prevents outward leaning of S6 (Figure 6B, left). In addition, S6 cyto $_{\text {. }}$ restricts movement of the U-motif toward the S2-S3 domain. In the open state of the WT, binding of $\mathrm{Ca}^{2+}$ moves CTD which transmits signals into two downstream pathways to open the channel; S4-S5 linker is unlocked by sequential conformational changes beginning at the U-motif and S6 leans outward by the movement of $\mathbf{S} 6_{\text {cyto. }}$. However, since $S 6_{\text {cyto }}$ still restricts the U-motif, the activity may be medium (Figure 6B, right). Mutations in the U-motif/S2-S3 domain, S1/S2, or S2/S3 interface disrupt the interaction which is essential for the channel opening (Figure 6C, left). Upon $\mathrm{Ca}^{2+}$ binding, conformational changes stop on the way, resulting in loss-of-function (Figure 6C, right). Mutations in S3/S4 or S1/S4 interface unlock the S4-S5 linker (Figure 6D, upper left). However, in the absence of $\mathrm{Ca}^{2+}$, this does not induce spontaneous outward movement of the S4-S5 linker and the channel pore kept closed. In the presence of $\mathrm{Ca}^{2+}$, these channels will show gain-of-function, since the S4-S5 linker is more mobile (Figure 6D, upper right). Mutations in the U-motif/S6 $6_{\text {cyto }}$ or the U-motif/CTD disrupt the U-motif/S6 $6_{\text {cyto }}$ interaction to make the U-motif and S6 $6_{\text {cyto }}$ more mobile (Figure 6D, lower left). Upon $\mathrm{Ca}^{2+}$ binding, these channels will show gain-of-function by an increased mobility in the S4-S5 linker and $S 6_{\text {cyto }}$ (Figure 6D, lower right). Considering that residues involved in the interactions are highly conserved and pathogenic mutations in RyR1 (L4505P, D4744H, and F4888Y; see Table 1) also showed corresponding behaviors, the fundamental mechanism of channel gating may be common among three RyR subtypes.

Outward movement of the S4-S5 linker is important in the channel opening, as it creates the space where S6 can lean into (des Georges et al., 2016; Peng et al., 2016). des Georges et al. (des Georges et al., 2016) provided a more detailed description about the movement of the S4-S5 linker in RyR1; it is bent in the direction of $\mathbf{S} 6$ in the closed state, but it straightens and alters its contacts 
with S6, thereby opening the channel pore. We showed similar conformational changes in the S4-S5 linker in RyR2 and further demonstrated that rewinding of the upper part of S4 to an a-helix, which is caused by rotation of the S1-S4 bundle, drives the S4-S5 linker movement (Figure 3H-3J and S4DS4F). Regulatory mechanisms of interaction between the S4-S5 linker and S6 will be the next subject for complete understanding of channel gating.

In RyR1, rearrangement of the salt bridges between S1 (R4563), S3 (Y4791) and S4 (D4815) has been reported to occur upon channel opening (des Georges et al., 2016). We showed that the corresponding salt bridges between R4501, Y4720, and D4744 were preserved in RyR2 but maintained in both closed and open states (Figure 3A-3C and S4A-S4C). Functional assays revealed that hydrogen bonds/salt bridges are critical to keep the stopper in place, as disruption of salt bridges by alanine substitutions or pathogenic mutations caused severe gain-of-function of the channel (Figure 3D-3G and S4G). Interestingly, a recent study showed that diamide insecticides bind to the pocket within the S1-S4 bundle of RyR1, thereby disrupting the salt bridge and opening RyR1 (Ma et al., 2020). Our results reasonably explain why such the insecticides activate the RyR channel.

The U-motif is proposed to be important in channel gating as it forms a stable complex with S6 $6_{\text {cyto }}$ and CTD, which move together when the channel opens (Yan et al., 2015). This movement is accompanied by a rigid shift in the S2-S3 domain, which has been proposed to eventually open the channel pore (Bai et al., 2016). We found that interaction of the U-motif with the S2-S3 domain is critically important in the channel opening; disruption of the interaction causes loss-of-function of the channel (Figure 2 and S3). In addition, we demonstrated that the U-motif/S6 $6_{\text {cyto }}$ interaction stabilizes the channel to the closed state (Figure 4 and S5). Stabilization of the channel is important not only for prevention of the hyperactivity but also for regulation by various stimuli or modifications that activate the channel, e.g., phosphorylation or oxidation/S-nitrosylation (Benkusky et al., 2004; Santulli et al., 2018). The U-motif/S6 $6_{\text {cyto }}$ interaction is the stabilization mechanism first identified at the molecular level and thus may contribute to regulation of the RyR channel.

In RyRs, the large cytoplasmic region is believed to play an important role in regulating channel opening. Indeed, a number of pathogenic mutations is located in the cytoplasmic region. The question arises as to how these mutations, located far from the core domain, cause changes in the channel activity. We demonstrated that the C-Central domain forms a tight complex with the $U$ motif/S6 cyto/CTD to move together upon $\mathrm{Ca}^{2+}$ binding (Figures $1 \mathrm{~F}, \mathrm{~S} 2 \mathrm{E}$, and S2F). The N-Central domain also moves upon $\mathrm{Ca}^{2+}$ binding (Figures $1 \mathrm{~A}$ and $1 \mathrm{~F}$ ). Through the $\mathrm{N}-/ \mathrm{C}$-Central domains, the core domain has a series of interactions with the N-terminal domains (NTD), HANDLE, and HD1 domains which consist a major part of the large cytoplasmic region (Peng et al., 2016; Yan et al., 
2015). Thus, it is possible that the conformational changes of the cytoplasmic region by pathogenic mutations affect the channel gating through these interactions. Recently, RyR1 and RyR2 structures carrying gain-of-function mutations at N-terminal domains (NTD) were reported (lyer et al., 2020). Determining the structures of various mutant RyRs will help to elucidate the long-range allosteric gating mechanism in the future.

In conclusion, we have revealed the gating mechanism of RyR2 upon $\mathrm{Ca}^{2+}$ binding and provided structural insights into the effects of pathogenic mutations on channel activity. These findings may greatly help develop more effective drugs to treat RyR2-associated diseases.

\section{Acknowledgements}

We thank Mariko Kurakata for assistance with cell culture. We thank Ikue Hiraga and the Laboratory of Radioisotope Research, Research Support Center, Juntendo University Graduate School of Medicine, for technical assistance. We also thank the staff scientists at the University of Tokyo's cryoEM facility. This study was partly supported by the Japan Society for the Promotion of Sciences KAKENHI (grant number JP16H04748 to H.O., 19 K07105 to N.K., and $19 H 03404$ to T.M.); the Platform Project for Supporting Drug Discovery and Life Science Research (Basis for Supporting Innovative Drug Discovery and Life Science Research [BINDS]; grant number JP20am0101080 to H.O. and T.M.); the Practical Research Project for Rare/Intractable Diseases from the Japan Agency for Medical Research and Development (AMED; grant number 19ek0109202) to N.K.); an Intramural Research Grant (29-4 and 2-5) for Neurological and Psychiatric Disorders of NCNP (to T.M.); and the Vehicle Racing Commemorative Foundation (to H.O. and T.M.).

\section{Author contributions}

T.K., N.K., T.M., and H.O. conceived and designed the project. M.Ko. and H.O. performed cell culture. T.K. performed protein purification. K.S. performed negative-staining EM studies. A.T. and T.K. prepared the grid for cryo-EM. A.T. and M.Ki. processed the images. H.O. performed model building and refinement. T.M. and N.K. performed the functional analysis. T.K., A.T., N.K., K.S., M.Ko., T.S. M.Ki., T.M., and H.O interpreted the data. T.M. and H.O supervised the project. H.O. and T.M. wrote the manuscript with input from all authors.

\section{Data and Code Availability}

Atomic coordinates and cryo-EM density maps have been deposited in the PDB and the Electron Microscopy Data Bank under the accession codes 7FJ6 and EMD-30688 (closed state before 
classification), 7FJ7 and EMD-30689 (closed state class1), 7FJ8 and EMD-30690 (closed state class2), 7FJ9 and EMD-30691 (open state class1), 7FJA and EMD-30692 (open state class2), 7FJB and EMD-30693 (open state class3), 7FJD and EMD-30694 (closed-like F4749A mutant in the presence of EGTA), 7FJE and EMD-30695 (open-like F4749A mutant in the presence of EGTA), 7FJF and EMD-30696 (F4749A mutant in the presence of $100 \mu \mathrm{M}$ of $\mathrm{Ca}^{2+}$ ), 7FJG and EMD-30697 (closed-like F4888A in the presence of EGTA), and 7FJH and EMD-30698 (open-like F4888A in the presence of EGTA).

\section{Declaration of interests}

The authors declare no competing interests.

\section{References}

Adams, P.D., Afonine, P.V., Bunkoczi, G., Chen, V.B., Davis, I.W., Echols, N., Headd, J.J., Hung, L.W., Kapral, G.J., Grosse-Kunstleve, R.W., et al. (2010). PHENIX: a comprehensive Python-based system for macromolecular structure solution. Acta Crystallogr D 66, 213-221.

Afonine, P.V., Poon, B.K., Read, R.J., Sobolev, O.V., Terwilliger, T.C., Urzhumtsev, A., and Adams, P.D. (2018). Real-space refinement in PHENIX for cryo-EM and crystallography. Acta Crystallogr $D$ 74, 531-544.

Bagattin, A., Veronese, C., Bauce, B., Wuyts, W., Settimo, L., Nava, A., Rampazzo, A., and Danieli, G.A. (2004). Denaturing HPLC-based approach for detecting RYR2 mutations involved in malignant arrhythmias. Clin Chem 50, 1148-1155.

Bai, X.C., Yan, Z., Wu, J.P., Li, Z.Q., and Yan, N. (2016). The Central domain of RyR1 is the transducer for long-range allosteric gating of channel opening. Cell Res 26, 995-1006.

Benkusky, N.A., Farrell, E.F., and Valdivia, H.H. (2004). Ryanodine receptor channelopathies. Biochem Biophys Res Commun 322, 1280-1285.

Bers, D.M. (2004). Macromolecular complexes regulating cardiac ryanodine receptor function. J Mol Cell Cardiol 37, 417-429.

Bharucha-Goebel, D.X., Santi, M., Medne, L., Zukosky, K., Dastgir, J., Shieh, P.B., Winder, T., Tennekoon, G., Finkel, R.S., Dowling, J.J., et al. (2013). Severe congenital RYR1-associated myopathy: the expanding clinicopathologic and genetic spectrum. Neurology 80, 1584-1589.

Brünger, A.T., Adams, P.D., Clore, G.M., DeLano, W.L., Gros, P., Grosse-Kunstleve, R.W., Jiang, J.S., Kuszewski, J., Nilges, M., Pannu, N.S., et al. (1998). Crystallography \& NMR system: A new software suite for macromolecular structure determination. Acta Crystallogr D 54, 905-921. 
Cabra, V., Murayama, T., and Samsó, M. (2016). Ultrastructural analysis of self-associated RyR2s. Biophys J 110, 2651-2662.

Chi, X., Gong, D., Ren, K., Zhou, G., Huang, G., Lei, J., Zhou, Q., and Yan, N. (2019). Molecular basis for allosteric regulation of the type 2 ryanodine receptor channel gating by key modulators. Proc Natl Acad Sci U S A 116, 25575-25582.

Choi, G., Kopplin, L.J., Tester, D.J., Will, M.L., Haglund, C.M., and Ackerman, M.J. (2004). Spectrum and frequency of cardiac channel defects in swimming-triggered arrhythmia syndromes. Circulation 110, 2119-2124.

Clarke, O.B., and Hendrickson, W.A. (2016). Structures of the colossal RyR1 calcium release channel. Curr Opin Struct Biol 39, 144-152.

des Georges, A., Clarke, O.B., Zalk, R., Yuan, Q., Condon, K.J., Grassucci, R.A., Hendrickson, W.A., Marks, A.R., and Frank, J. (2016). Structural basis for gating and activation of RyR1. Cell 167, 145157.e117.

Eletr, S., and Inesi, G. (1972). Phospholipid orientation in sarcoplasmic membranes: spin-label ESR and proton MNR studies. Biochim Biophys Acta 282, 174-179.

Emsley, P., Lohkamp, B., Scott, W.G., and Cowtan, K. (2010). Features and development of Coot. Acta Crystallogr D 66, 486-501.

Endo, M. (2009). Calcium-induced calcium release in skeletal muscle. Physiol Rev 89, 1153-1176.

Fabiato, A., and Fabiato, F. (1978). Calcium-induced release of calcium from the sarcoplasmic reticulum of skinned cells from adult human, dog, cat, rabbit, rat, and frog hearts and from fetal and new-born rat ventricles. Ann NY Acad Sci 307, 491-522.

Fujii, Y., Itoh, H., Ohno, S., Murayama, T., Kurebayashi, N., Aoki, H., Blancard, M., Nakagawa, Y., Yamamoto, S., Matsui, Y., et al. (2017). A type 2 ryanodine receptor variant associated with reduced $\mathrm{Ca}^{2+}$ release and short-coupled torsades de pointes ventricular arrhythmia. Heart Rhythm 14, 98-107. Gong, D.S., Chi, X.M., Wei, J.H., Zhou, G.W., Huang, G.X.Y., Zhang, L., Wang, R.W., Lei, J.L., Chen, S.R.W., and Yan, N. (2019). Modulation of cardiac ryanodine receptor 2 by calmodulin. Nature 572, 347-351.

Hayashi, M., Denjoy, I., Extramiana, F., Maltret, A., Buisson, N.R., Lupoglazoff, J.M., Klug, D., Hayashi, M., Takatsuki, S., Villain, E., et al. (2009). Incidence and risk factors of arrhythmic events in catecholaminergic polymorphic ventricular tachycardia. Circulation 119, 2426-2434.

Ibarra M, C.A., Wu, S., Murayama, K., Minami, N., Ichihara, Y., Kikuchi, H., Noguchi, S., Hayashi, Y.K., Ochiai, R., and Nishino, I. (2006). Malignant hyperthermia in Japan: mutation screening of the 
entire ryanodine receptor type 1 gene coding region by direct sequencing. Anesthesiology $104,1146-$ 1154.

Iyer, K.A., Hu, Y., Nayak, A.R., Kurebayashi, N., Murayama, T., and Samso, M. (2020). Structural mechanism of two gain-of-function cardiac and skeletal RyR mutations at an equivalent site by cryoEM. Sci Adv 6, eabb2964.

Jiang, D., Chen, W., Wang, R., Zhang, L., and Chen, S.R. (2007). Loss of luminal $\mathrm{Ca}^{2+}$ activation in the cardiac ryanodine receptor is associated with ventricular fibrillation and sudden death. Proc Natl Acad Sci U S A 104, 18309-18314.

Kawamura, M., Ohno, S., Naiki, N., Nagaoka, I., Dochi, K., Wang, Q., Hasegawa, K., Kimura, H., Miyamoto, A., Mizusawa, Y., et al. (2013). Genetic background of catecholaminergic polymorphic ventricular tachycardia in Japan. Circ J 77, 1705-1713.

Ma, R., Haji-Ghassemi, O., Ma, D., Jiang, H., Lin, L., Yao, L., Samurkas, A., Li, Y., Wang, Y., Cao, P., et al. (2020). Structural basis for diamide modulation of ryanodine receptor. Nat Chem Biol 16, 1246-1254.

Mastronarde, D.N. (2005). Automated electron microscope tomography using robust prediction of specimen movements. J Struct Biol 152, 36-51.

Medeiros-Domingo, A., Bhuiyan, Z.A., Tester, D.J., Hofman, N., Bikker, H., van Tintelen, J.P., Mannens, M.M., Wilde, A.A., and Ackerman, M.J. (2009). The RYR2-encoded ryanodine receptor/calcium release channel in patients diagnosed previously with either catecholaminergic polymorphic ventricular tachycardia or genotype negative, exercise-induced long QT syndrome: a comprehensive open reading frame mutational analysis. J Am Coll Cardiol 54, 2065-2074.

Meissner, G. (1994). Ryanodine receptor/ $\mathrm{Ca}^{2+}$ release channels and their regulation by endogenous effectors. Annu Rev Physiol 56, 485-508.

Murayama, T., Kurebayashi, N., Ishigami-Yuasa, M., Mori, S., Suzuki, Y., Akima, R., Ogawa, H., Suzuki, J., Kanemaru, K., Oyamada, H., et al. (2018a). Efficient high-throughput screening by endoplasmic reticulum $\mathrm{Ca}^{2+}$ measurement to identify inhibitors of ryanodine receptor $\mathrm{Ca}^{2+}$-release channels. Mol Pharmacol 94, 722-730.

Murayama, T., Ogawa, H., Kurebayashi, N., Ohno, S., Horie, M., and Sakurai, T. (2018b). A tryptophan residue in the caffeine-binding site of the ryanodine receptor regulates $\mathrm{Ca}^{2+}$ sensitivity. Commun Biol 1, 98.

Nozaki, Y., Kato, Y., Uike, K., Yamamura, K., Kikuchi, M., Yasuda, M., Ohno, S., Horie, M., Murayama, T., Kurebayashi, N., et al. (2020). Co-phenotype of left ventricular non-compaction cardiomyopathy 
and atypical catecholaminergic polymorphic ventricular tachycardia in association with R169Q, a ryanodine receptor type 2 missense mutation. Circ J 84, 226-234.

Ogawa, H., Kurebayashi, N., Yamazawa, T., and Murayama, T. (2020). Regulatory mechanisms of ryanodine receptor/ $\mathrm{Ca}^{2+}$ release channel revealed by recent advancements in structural studies. $\mathrm{J}$ Muscle Res Cell Motil.

Ogawa, Y. (1994). Role of ryanodine receptors. Crit Rev Biochem Mol Biol 29, 229-274.

Ohno, S., Omura, M., Kawamura, M., Kimura, H., Itoh, H., Makiyama, T., Ushinohama, H., Makita, N., and Horie, M. (2014). Exon 3 deletion of RYR2 encoding cardiac ryanodine receptor is associated with left ventricular non-compaction. Europace 16, 1646-1654.

Ozawa, J., Ohno, S., Fujii, Y., Makiyama, T., Suzuki, H., Saitoh, A., and Horie, M. (2018). Differential diagnosis between catecholaminergic polymorphic ventricular tachycardia and long QT syndrome type 1- modified schwartz score. Circ J 82, 2269-2276.

Paech, C., Gebauer, R.A., Karstedt, J., Marschall, C., Bollmann, A., and Husser, D. (2014). Ryanodine receptor mutations presenting as idiopathic ventricular fibrillation: a report on two novel familial compound mutations, c.6224T>C and c. $13781 \mathrm{~A}>\mathrm{G}$, with the clinical presentation of idiopathic ventricular fibrillation. Pediatr Cardiol 35, 1437-1441.

Peng, W., Shen, H.Z., Wu, J.P., Guo, W.T., Pan, X.J., Wang, R.W., Chen, S.R.W., and Yan, N. (2016). Structural basis for the gating mechanism of the type 2 ryanodine receptor RyR2. Science 354 .

Pérez-Riera, A.R., Barbosa-Barros, R., de Rezende Barbosa, M.P.C., Daminello-Raimundo, R., de Lucca, A.A., Jr., and de Abreu, L.C. (2018). Catecholaminergic polymorphic ventricular tachycardia, an update. Ann Noninvas Electrocardiol 23, e12512.

Pettersen, E.F., Goddard, T.D., Huang, C.C., Couch, G.S., Greenblatt, D.M., Meng, E.C., and Ferrin, T.E. (2004). UCSF Chimera--a visualization system for exploratory research and analysis. J Comput Chem 25, 1605-1612.

Priori, S.G., and Chen, S.R. (2011). Inherited dysfunction of sarcoplasmic reticulum Ca2+ handling and arrhythmogenesis. Circ Res 108, 871-883.

Priori, S.G., Napolitano, C., Memmi, M., Colombi, B., Drago, F., Gasparini, M., DeSimone, L., Coltorti, F., Bloise, R., Keegan, R., et al. (2002). Clinical and molecular characterization of patients with catecholaminergic polymorphic ventricular tachycardia. Circulation 106, 69-74.

Rohou, A., and Grigorieff, N. (2015). CTFFIND4: fast and accurate defocus estimation from electron micrographs. J Struct Biol 192, 216-221.

Roston, T.M., Haji-Ghassemi, O., LaPage, M.J., Batra, A.S., Bar-Cohen, Y., Anderson, C., Lau, Y.R., Maginot, K., Gebauer, R.A., Etheridge, S.P., et al. (2018). Catecholaminergic polymorphic ventricular 
tachycardia patients with multiple genetic variants in the PACES CPVT Registry. PLOS ONE 13, e0205925.

Santulli, G., Lewis, D., des Georges, A., Marks, A.R., and Frank, J. (2018). Ryanodine Receptor Structure and Function in Health and Disease. Subcell Biochem 87, 329-352.

Shigemizu, D., Aiba, T., Nakagawa, H., Ozaki, K., Miya, F., Satake, W., Toda, T., Miyamoto, Y., Fujimoto, A., Suzuki, Y., et al. (2015). Exome analyses of long QT syndrome reveal candidate pathogenic mutations in calmodulin-interacting genes. PLOS ONE 10, e0130329.

Smart, O.S., Goodfellow, J.M., and Wallace, B.A. (1993). The pore dimensions of gramicidin A. Biophys J 65, 2455-2460.

Suzuki, J., Kanemaru, K., Ishii, K., Ohkura, M., Okubo, Y., and lino, M. (2014). Imaging intraorganellar $\mathrm{Ca}^{2+}$ at subcellular resolution using CEPIA. Nat Commun 5, 4153.

Tang, Y., Tian, X., Wang, R., Fill, M., and Chen, S.R. (2012). Abnormal termination of $\mathrm{Ca}^{2+}$ release is a common defect of RyR2 mutations associated with cardiomyopathies. Circ Res 110, 968-977.

Tester, D.J., Spoon, D.B., Valdivia, H.H., Makielski, J.C., and Ackerman, M.J. (2004). Targeted mutational analysis of the RyR2-encoded cardiac ryanodine receptor in sudden unexplained death: a molecular autopsy of 49 medical examiner/coroner's cases. Mayo Clin Proc 79, 1380-1384.

Uehara, A., Murayama, T., Yasukochi, M., Fill, M., Horie, M., Okamoto, T., Matsuura, Y., Uehara, K., Fujimoto, T., Sakurai, T., et al. (2017). Extensive $\mathrm{Ca}^{2+}$ leak through $\mathrm{K} 4750 \mathrm{Q}$ cardiac ryanodine receptors caused by cytosolic and luminal $\mathrm{Ca}^{2+}$ hypersensitivity. J Gen Physiol 149, 199-218.

Wang, D., Shah, K.R., Um, S.Y., Eng, L.S., Zhou, B., Lin, Y., Mitchell, A.A., Nicaj, L., Prinz, M., McDonald, T.V., et al. (2014). Cardiac channelopathy testing in 274 ethnically diverse sudden unexplained deaths. Forensic Sci Int 237, 90-99.

Wong, L.C., Roses-Noguer, F., Till, J.A., and Behr, E.R. (2014). Cardiac evaluation of pediatric relatives in sudden arrhythmic death syndrome: a 2-center experience. Circ Arrhythm Electrophysiol 7, 800-806.

Wu, S., Ibarra, M.C., Malicdan, M.C., Murayama, K., Ichihara, Y., Kikuchi, H., Nonaka, I., Noguchi, S., Hayashi, Y.K., and Nishino, I. (2006). Central core disease is due to RYR1 mutations in more than $90 \%$ of patients. Brain 129, 1470-1480.

Yan, Z., Bai, X.C., Yan, C.Y., Wu, J.P., Li, Z.Q., Xie, T., Peng, W., Yin, C.C., Li, X.M., Scheres, S.H.W., et al. (2015). Structure of the rabbit ryanodine receptor RyR1 at near-atomic resolution. Nature 517, 50-55.

Zalk, R., and Marks, A.R. (2017). $\mathrm{Ca}^{2+}$ release channels join the "resolution revolution". Trends Biochem Sci 42, 543-555. 
Zhao, Y., Araki, S., Wu, J., Teramoto, T., Chang, Y.F., Nakano, M., Abdelfattah, A.S., Fujiwara, M., Ishihara, T., Nagai, T., et al. (2011). An expanded palette of genetically encoded $\mathrm{Ca}^{2+}$ indicators. Science 333, 1888-1891.

Zivanov, J., Nakane, T., Forsberg, B.O., Kimanius, D., Hagen, W.J., Lindahl, E., and Scheres, S.H. (2018). New tools for automated high-resolution cryo-EM structure determination in RELION-3. eLife

7.

\section{Figure legends}

Fig. 1. Conformational changes upon $\mathrm{Ca}^{2+}$ binding. (A) Overlay of RyR2 in the closed (light blue) and open (yellow) states viewed from the direction parallel to the lipid bilayer and shown as a ribbon model. Two facing protomers in the RyR2 tetramer are shown. (B) Magnified view of the dotted box in (A). In the left protomer, each domain is colored (N-central, light pink; C-central, purple; U-motif, magenta; S1-S5, wheat; S2-S3 domain, light green; S4-S5 linker, warm pink; S6, blue; CTD, orange). S4-S5 linker and S6 moved outside upon $\mathrm{Ca}^{2+}$ binding as indicated by the red arrows. Three regions parallel to the membrane are defined as CTD, U-motif, and S4-S5 layers. $\mathrm{Ca}^{2+}$, shown as cyan ball; $\mathrm{Zn}^{2+}$, shown as gray ball. (C-E) Cross-section views of CTD, U-motif, and S4-S5 layers. Closed state is colored in light blue and open state is colored according to $(B)$ or yellow. In (E), Ca representation overlaid with cylindrical TM helices are used. $\mathrm{Ca}^{2+}$ binding causes clockwise rotation of CTD (green arrow in C), U-motif (green arrow in D), and S1-S4 TM helices and outward movement of S4-S5 linker

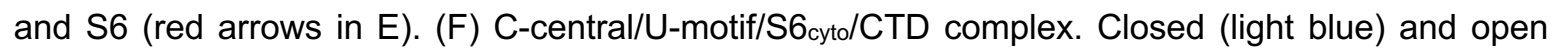
(colored according to B) states are overlaid at the CTD. Central domain is split into two parts at G3987 as the pivot upon $\mathrm{Ca}^{2+}$ binding. $(\mathrm{G})$ Rotation of the $\mathrm{C}$-central/U-motif/S6 ${ }_{\text {cyto }} / \mathrm{CTD}$ complex upon $\mathrm{Ca}^{2+}$ binding viewed from the rotation axis. (H) Scheme of channel opening upon $\mathrm{Ca}^{2+}$ binding. Two independent pathways via S6 and S4-S5 linker are hypothesized.

Fig. 2. Key interactions between U-motif and S2-S3 domain upon channel opening. (A-C) Interface of U-motif and S2-S3 domain in the closed state (A), open state (B), and an overlay of both states $(C)$ viewed parallel to the membrane and shown as a Ca model. Amino acid residues involved in key interactions are shown as stick models. The color of carbon atoms is the same as that of Ca; oxygen, red; nitrogen, blue. The TM region is indicated in yellow and the region forming a-helices is overlaid with the cylinder model. Hydrogen bonds or salt bridges are shown as orange dotted lines. Density maps around side chains shown in (A) and (B) are superimposed and contoured at 0.025 . (D-G) Functional analysis of mutants involved in U-motif/S2-S3 domain interaction. (D) $\mathrm{Ca}^{2+}-$ 
dependent $\left[{ }^{3} \mathrm{H}\right]$ ryanodine binding of WT and representative mutants. (E) Summary of $\left[{ }^{3} \mathrm{H}\right] \mathrm{ryanodine}$ binding of WT and mutants at pCa 4.5. (F) Representative traces of cytoplasmic $\left(\left[\mathrm{Ca}^{2+}\right]_{\mathrm{cyt}}\right)$ and ER ([Ca $\left.{ }^{2+}\right]$ ER) $\mathrm{Ca}^{2+}$ signals of HEK293 cells expressing WT or K4593A. Spontaneous $\mathrm{Ca}^{2+}$ oscillations occurred with a concomitant decrease in $\left[\mathrm{Ca}^{2+}\right]_{E R}$ in WT, while the $\mathrm{K} 4593$ mutant showed no $\mathrm{Ca}^{2+}$ oscillations with an increased $\left[\mathrm{Ca}^{2+}\right] \mathrm{ER}$, indicating loss-of-function of the channel. (G) Summary of upper level of $\left[\mathrm{Ca}^{2+}\right]_{E R}$ signals in WT and mutants. All mutants showed loss-of-function of the channel. Data are presented as the mean $\pm \mathrm{SD}$. ${ }^{*} p<0.05$; ${ }^{* *} p<0.01$; ${ }^{* * *} p<0.001$. (H) Multiple sequence alignment of three RyR isoforms around the U-motif, S1, and S2 to the initial S2-S3 domain. The gray-shaded residues are the identical sequences among isoforms, and the residues in which mutants were prepared and functional assayed are red-shaded. Secondary structures are shown above the alignment. $m$, mouse; $h$, human; $p$, pig; $r$, rabbit.

Fig. 3. Key interactions in the transmembrane region upon channel opening. (A-C) The S1-S4 bundle. Closed state $(A)$, open state $(B)$, and overlay of the structures in both states $(C)$ are shown as a Ca model and overlaid with cylinder models. Hydrogen bonds/salt bridges are shown as orange botted lines. Density maps around side chains shown in (A) and (B) are superimposed and contoured at 0.03. (D-G) Functional analysis of mutants involved in the S1/S2, S2/S3, S1/S4, or S3/S4 interaction. (D) $\mathrm{Ca}^{2+}$-dependent $\left[{ }^{3} \mathrm{H}\right]$ ryanodine binding of $W T$ and representative mutants. (E) Summary of $\left[{ }^{3} \mathrm{H}\right]$ ryanodine binding of WT and mutants at pCa 4.5. (F) Representative traces of $\left[\mathrm{Ca}^{2+}\right]_{\mathrm{cyt}}$ and $\left[\mathrm{Ca}^{2+}\right]_{E R}$ signals of HEK293 cells expressing Y4589A or D4744A. (G) Summary of upper level of $\left[\mathrm{Ca}^{2+}\right]_{E R}$ signals in WT and mutants. (H-J) TM region around $\mathrm{S} 4$. Closed state $(\mathrm{H})$, open state (I), and overlay of the structures in both states $(\mathrm{J})$. Structures in both states were fitted to the bottom part of S4 helices and viewed along the axis of the S4 helix and from the cytoplasm; Ca model overlaid with cylinder models. Main chain representation of the S4-S5 linker. Density maps around side chains shown in $(\mathrm{H})$ and $(\mathrm{I})$ are superimposed and contoured at 0.03 . $(\mathrm{K}) \mathrm{Ca}^{2+}$-dependent $\left[{ }^{3} \mathrm{H}\right]$ ryanodine binding of WT and representative mutants. (L) Summary of $\left[{ }^{3} \mathrm{H}\right]$ ryanodine binding of WT and mutants at $\mathrm{pCa}$ 4.5. (M) Representative traces of $\left[\mathrm{Ca}^{2+}\right]_{c y t}$ and $\left[\mathrm{Ca}^{2+}\right]_{\mathrm{ER}}$ signals of $\mathrm{HEK} 293$ cells expressing L4505A or F4749A. (N) Summary of upper level of $\left[\mathrm{Ca}^{2+}\right]_{E R}$ signals in WT and mutants. (O) Multiple sequence alignment of three RyR isoforms around S1, S2, and S3 to S4. The gray-shaded residues are the identical sequences among isoforms, and the residues in which mutants were prepared and functional assayed are red-shaded. Secondary structures are shown above the alignment. m, mouse; h, human; $p$, pig; r, rabbit. 
Fig. 4. Key interactions between the U-motif and S6 cyto/CTD. (A) Structure around the U-motif in

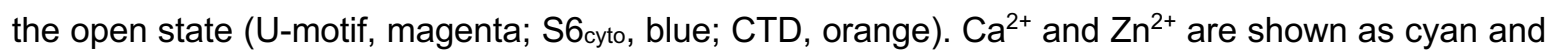
gray spheres, respectively. (B, C) Details of the U-motif/S6 cyto interaction. Structures in the closed (B) and open $(\mathrm{C})$ states fitted to the N-terminal region of the U-motif are shown as a full atomic model. Density maps around the interaction are superimposed and contoured at 0.025 . (D) Details of the Umotif/CTD interaction around F4888. Overly of the structures in the closed (light blue) and open (colored) states. (E-L) Functional analysis of mutants involved in U-motif/S6cyto $(E-H)$ and $U$ motif/CTD (I-L) interactions. (E, I) $\mathrm{Ca}^{2+}$-dependent $\left[{ }^{3} \mathrm{H}\right]$ ryanodine binding of WT and representative mutants. (F, J) Summary of $\left[{ }^{3} \mathrm{H}\right]$ ryanodine binding of $W T$ and mutants at pCa 4.5. (G, K) Representative traces of $\left[\mathrm{Ca}^{2+}\right]_{\mathrm{cyt}}$ and $\left[\mathrm{Ca}^{2+}\right]_{E R}$ signals of $\mathrm{HEK} 293$ cells expressing F4173A or V4879A $(\mathrm{G})$ and F4888A or V4175A (K). (H, L) Summary of upper level of $\left[\mathrm{Ca}^{2+}\right]$ ER signals in WT and mutants. (M) Multiple sequence alignment of three RyR isoforms around the Umotif and S6 cyto to the CTD. The gray-shaded residues are the identical sequences among isoforms, and the residues in which mutants were prepared and functional assayed are red-shaded. Secondary structures are shown above the alignment. $m$, mouse; $h$, human; $p$, pig; $r$, rabbit.

Fig. 5. Structural basis of gain-of-function mutations and gating mechanism upon $\mathrm{Ca}^{2+}$ binding. (A) Structure of closed-like F4888A (colored) overlaid with WT in the closed state (light blue). (B) Enlarged view of the dotted box in (A) superposed with density maps contoured at 0.025 . Magenta and green nets represent density maps around WT U-motif in the closed state and closed-like F4888A, respectively. Light blue and blue nets represent density maps around WT S6 6 cyto in the closed state and closed-like F4888A, respectively. (C) Overlay of closed-like F4749A (orange) and WT in the closed state (light blue) at the TM region viewed from the cytoplasm. (D) Interface of U-motif and S2S3 domain of open-like F4888A (colored) overlaid with WT in the open state (yellow) and in the closed state (only U-motif is shown, blue). Note that in the mutant, parallel movement of the U-motif and S2S3 domains toward the outside compared with the WT in the open state is completely different from the rotation motion seen in the WT structural change from closed to open state. (E) Overlay of the TM region of open-like F4888A (violet) with WT in the open state (yellow). (F) Details of the Umotif/S6 cyto interaction. Open-like F4888A and WT in the open states fitted to the N-terminal region of the U-motif are superposed with density maps contoured at 0.025 . Magenta and light pink nets represent density maps around the WT U-motif in the open state and open-like F4888A, respectively. Light blue and orange nets represent density maps around WT S6 $6_{\text {cyto }}$ in the open state and open-like F4888A, respectively. $(G, H)$ Scheme of the structure in the closed $(G)$ and open $(H)$ state. In the 
closed state, the upper part of S4 does not form an a-helix. The S4-S5 linker is unfolded and significantly bends in the direction of S6. Binding of $\mathrm{Ca}^{2+}$ to the C-central/CTD interface causes $9.8^{\circ}$ rotation of the C-central/U-motif/S6yto/CTD complex, leading to two pathways. Pathway 1 : the rotation causes $30^{\circ}$ rotation of $S 6_{\text {cyto }}$ which loosens the U-motif/S6 $6_{\text {cyto }}$ interaction and allows outward movement of S6. Pathway 2: a sequential movement of the S2S3 domain, S2, S1-S4 bundle, and S4 allows the upper part of S4 to rewind and form an a-helix. Subsequently, the S4-S5 linker moves outward, creating a space where $\mathrm{S} 6$ can lean into. A combination of these two independent pathways eventually leads to opening of the channel.

Fig. 6. Details of interactions and schematic diagram of the RyR2 channel gating mechanism upon $\mathrm{Ca}^{2+}$ binding. (A) Details of interactions identified in this study. Amino-acid residues shown in red letter and blue letter indicate gain-of-function and loss-of-function by alanine-substituted or pathogenic mutations, respectively. Arrows indicate interactions. Yellow and dark blue arrows indicate interactions only found in the closed and open states, respectively. (B-D) Schematic diagram of the RyR2 channel gating mechanism upon $\mathrm{Ca}^{2+}$ binding. The left and right diagrams show the states in the absence and presence of $\mathrm{Ca}^{2+}$, respectively. The black lines, the domain interactions; The blue T-shaped lines, the domain interactions that act as the suppression; The black lines with arrowhead, the activated domain interactions. The domains shown in gray-scale and colored indicate the domains in the inactive and active states, respectively. (B) Wild type (WT). Since the movement of S4-S5 linker is locked, the channel pore is closed. $\mathrm{Ca}^{2+}$ binding unlocks the S4-S5 linker and induces the outward leaning of S6, resulting in the pore opening. (C) Loss-of-function (LOF) mutants. Mutations in the U-motif/S2-S3 domain (\#1), S1/S2 (\#2) or S2/S3 (\#3) interface cause disconnection of signal transduction. Binding of $\mathrm{Ca}^{2+}$ therefore cannot induce the outward movement of the S4-S5 linker and the channel pore is kept closed. (D) Gain-of-function (GOF) mutants. (Upper panels) Mutations in S3/S4 (\#4) or S1/S4 (\#5) interface unlock the S4-S5 linker to be activated. In the absence of $\mathrm{Ca}^{2+}$, the channel pore is kept closed, since the outward leaning of $\mathrm{S} 6$ does not occur spontaneously. Binding of $\mathrm{Ca}^{2+}$ causes hyperactivity of the channel, since the S4-S5 linker is more active than WT. (Lower panels) Mutations in U-motif, S6 $6_{\text {cyto }}$ or CTD (\#6) reduce or lose U-motif/S6 $6_{\text {cyto }}$ interaction. Binding of $\mathrm{Ca}^{2+}$ causes hyperactivity of the channel, since S6 and the S4-S5 linker is more active than WT. 
Table 1. RyR2 mutants used in this study.

\begin{tabular}{|c|c|c|c|c|c|c|}
\hline Mutation & Domain & Disease & Ref & $\begin{array}{c}\text { Ryanodine } \\
\text { binding }\end{array}$ & $\begin{array}{c}\mathrm{ER} \mathrm{Ca}^{2+} \\
\text { level }\end{array}$ & $\begin{array}{c}\text { Functional } \\
\text { change }\end{array}$ \\
\hline S4167A & U-motif & & & $\downarrow$ & $\uparrow$ & LOF \\
\hline S4167P & U-motif & CPVT (RyR2) & (Ozawa et al., 2018) & $\downarrow$ & $\uparrow$ & LOF \\
\hline $\mathrm{F} 4171 \mathrm{~A}$ & U-motif & & & $\uparrow$ & $\downarrow$ & GOF \\
\hline 14172A & U-motif & & & $\uparrow$ & $\downarrow$ & GOF \\
\hline F4173A & U-motif & & & $\uparrow$ & $\downarrow$ & GOF \\
\hline V4175A & U-motif & & & $\uparrow$ & $\downarrow$ & GOF \\
\hline V4176A & U-motif & & & $\uparrow$ & $\downarrow$ & GOF \\
\hline N4177A & U-motif & & & $\uparrow$ & $\downarrow$ & GOF \\
\hline N4177S & U-motif & CPVT (RyR2) & $\begin{array}{c}\text { (Medeiros-Domingo et } \\
\text { al., 2009) }\end{array}$ & $\uparrow$ & $\downarrow$ & GOF \\
\hline N4177Y & U-motif & CPVT (RyR2) & (Hayashi et al., 2009) & $\uparrow$ & $\downarrow$ & GOF \\
\hline E4193A & U-motif & & & $\downarrow$ & $\uparrow$ & LOF \\
\hline E4198A & U-motif & & & $\downarrow$ & $\uparrow$ & LOF \\
\hline F4497A & s1 & & & $\downarrow$ & $\uparrow$ & LOF \\
\hline F4497C & S1 & CPVT (RyR2) & (Choi et al., 2004) & $\downarrow$ & $\uparrow$ & LOF \\
\hline Y4498A & s1 & & & $\downarrow$ & $\uparrow$ & LOF \\
\hline R4501A & s1 & & & $\uparrow$ & $\downarrow$ & GOF \\
\hline L4505A & s1 & & & $\uparrow$ & $\downarrow$ & GOF \\
\hline L4505P & S1 & CCD (RyR1) & (Wu et al., 2006) & $\uparrow$ & $\downarrow$ & GOF \\
\hline Y4589A & S2 & & & $\downarrow$ & $\uparrow$ & LOF \\
\hline L4592A & S2 & & & $\downarrow$ & $\uparrow$ & LOF \\
\hline K4593A & S2-S3 & & & $\downarrow$ & $\uparrow$ & LOF \\
\hline K4593Q & S2-S3 & LQTS (RyR2) & (Shigemizu et al., 2015) & $\downarrow$ & $\uparrow$ & LOF \\
\hline K4593R & S2-S3 & IVF (RyR2) & (Paech et al., 2014) & $\downarrow$ & $\uparrow$ & LOF \\
\hline R4607A & S2-S3 & & & $\downarrow$ & $\uparrow$ & LOF \\
\hline R4607Q & S2-S3 & Sudden death (RyR2) & (Wong et al., 2014) & $\downarrow$ & $\uparrow$ & LOF \\
\hline R4607W & S2-S3 & Sudden death (RyR2) & (Wang et al., 2014) & $\downarrow$ & $\uparrow$ & LOF \\
\hline D4715A & S3 & & & $\downarrow$ & $\uparrow$ & LOF \\
\hline Y4720A & S3 & & & $\uparrow$ & $\downarrow$ & GOF \\
\hline Y4720C & S3 & CPVT (RyR2) & (Roston et al., 2018) & $\uparrow$ & $\downarrow$ & GOF \\
\hline D4744A & S4 & & & $\uparrow$ & $\downarrow$ & GOF \\
\hline D4744H & S4 & Myopathy (RyR1) & $\begin{array}{c}\text { (Bharucha-Goebel et al., } \\
\text { 2013) }\end{array}$ & $\uparrow$ & $\downarrow$ & GOF \\
\hline F4749A & S4 & & & $\uparrow$ & $\downarrow$ & GOF \\
\hline Q4875A & S6cyto & & & $\uparrow$ & $\downarrow$ & GOF \\
\hline V4879A & S6cyto & CPVT (RyR2) & (Bagattin et al., 2004) & $\uparrow$ & $\downarrow$ & GOF \\
\hline F4888A & CTD & & & $\uparrow$ & $\downarrow$ & GOF \\
\hline F4888Y & CTD & MH/CCD (RyR1) & (Ibarra M et al., 2006) & $\uparrow$ & $\downarrow$ & GOF \\
\hline
\end{tabular}


bioRxiv preprint doi: https://doi.org/10.1101/2020.11.30.401026; this version posted February 12, 2021. The copyright holder for this preprint (which was not certified by peer review) is the author/funder. All rights reserved. No reuse allowed without permission.

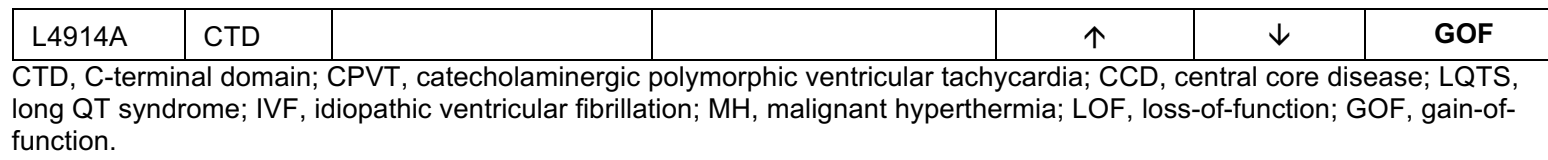




\section{Materials and methods}

\section{Mammalian cell lines and culture conditions}

HEK293 cells stably expressing mouse RyR2 (wild type or mutants) were grown in standard DMEM medium (GIBCO), supplemented with 10\% fetal bovine serum (FBS) (GIBCO), $100 \mathrm{U} / \mathrm{mL}$ penicillinstreptomycin (GIBCO), and maintained in a humidified incubator at $37^{\circ} \mathrm{C}$ with $5 \% \mathrm{CO}$. Growth and protein expression conditions are outlined in Method Details.

\section{Bacterial strains used for protein production}

Escherichia coli BL21 cells (Novagen) were used for the expression of the streptavidin-binding peptide (SBP)-tagged FKBP12.6. Growth and protein expression conditions are outlined in Method Details.

\section{Expression and purification of SBP-FKBP12.6}

Expression and purification of SBP-tagged FKBP12.6 was performed as described previously (Cabra et al., 2016) with some modifications. Briefly, cDNA for SBP-tagged human FKBP12.6 was subcloned into the pET28 vector (Novagen) containing a 6xHis-tag at the $\mathrm{N}$-terminus. Escherichia coli BL21 cells transformed with the above expression vector were grown at $37^{\circ} \mathrm{C}$ in $500 \mathrm{~mL}$ LB medium containing ampicillin at a final concentration of $50 \mathrm{mg} / \mathrm{L}$. After reaching an $\mathrm{OD}_{600}$ of $0.95-1.2$, SBPFKBP12.6 expression was induced by adding $1 \mathrm{mM}$ IPTG (Wako) for $3 \mathrm{~h}$ at $37^{\circ} \mathrm{C}$. Cells were then harvested, resuspended in $20 \mathrm{mM}$ MOPS-Na (pH 7.4), $300 \mathrm{mM} \mathrm{NaCl}, 20 \mathrm{mM}$ imidazole, and a cocktail of protease inhibitors [antipain (Peptide Institute), aprotinin (Nacalai), chymostatin (Peptide Institute), leupeptin (Peptide Institute) and pepstatin A (Peptide Institute), each $2 \mu \mathrm{g} / \mathrm{mL}$ ], and lysed using a sonicator in an ice bath. The lysate was centrifuged at $100,000 \times \mathrm{g}$ for $30 \mathrm{~min}$ at $4{ }^{\circ} \mathrm{C}$ and the pellet was discarded. The supernatant was incubated for $1 \mathrm{~h}$ at $4^{\circ} \mathrm{C}$ with $0.5 \mathrm{~mL}$ Profinity IMAC resin (BioRad Laboratories, Hercules, CA) in a buffer containing $20 \mathrm{mM}$ MOPS-Na (pH 7.4), $300 \mathrm{mM} \mathrm{NaCl}$, and $20 \mathrm{mM}$ imidazole. The resin was washed five times with the buffer and SBP-FKBP12.6 was eluted with buffer containing $300 \mathrm{mM}$ imidazole. The eluted protein was quickly frozen in liquid nitrogen and stored at $-80^{\circ} \mathrm{C}$. 


\section{Construction of mutant RyR2 cDNA expression vector and generation of stable HEK293 cell lines}

Alanine substitution or pathogenic mutations were introduced by inverse PCR using F5 cassette (nucleotide residue number 10,185-14,901 of mouse RyR2) as a template (Uehara et al., 2017). The mutations were confirmed by DNA sequencing. The mutant cassette was inserted into full-length wildtype mouse RyR2 expression vector (pcDNA5/FRT/TO-RyR2wt) (Uehara et al., 2017) by restriction enzymes (BsiW I/Not I). HEK293 cells stably expressing mutant RyR2 was generated using the FlpIn T-REx system (ThemoFisher) according to manufacturer's instructions. Clones with suitable expression of RyR2 were selected and used for experiments.

\section{Preparation of microsomes from RyR2-expressing HEK293 cells}

HEK293 cells stably expressing mouse RyR2 (wild type or mutants) were grown in 60 150-mm cell culture dishes. At $70-80 \%$ confluency, protein expression was induced by $2 \mu \mathrm{g} / \mathrm{mL}$ doxycycline (SIGMA) for $48 \mathrm{~h}$. Cells were then harvested, rinsed with cold phosphate-buffered saline (PBS) (GIBCO), and microsomes were prepared as described by Inesi et al. (Eletr and Inesi, 1972). Briefly, the cell pellet was resuspended in $60 \mathrm{~mL}$ of $10 \mathrm{mM} \mathrm{NaHCO}_{3}$ in presence of protease inhibitors and processed for nitrogen cavitation for $30 \mathrm{~min}$ at $1,000 \mathrm{psi}$. The suspension was diluted with $60 \mathrm{~mL}$ of $0.6 \mathrm{M}$ sucrose, $0.3 \mathrm{M} \mathrm{KCl}, 40 \mathrm{mM}$ MOPS-Na ( $\mathrm{pH}$ 7.4), and protease inhibitors and then centrifuged at $1,000 \times g$ for $10 \mathrm{~min}$. The supernatant was supplemented with $30 \mathrm{~mL}$ of $2.4 \mathrm{M} \mathrm{KCl}, 0.3 \mathrm{M}$ sucrose, $20 \mathrm{mM}$ MOPS-Na ( $\mathrm{pH} 7.4$ ), and the above protease inhibitor cocktail and centrifuged at 10,000 $\times g$ for $20 \mathrm{~min}$. The supernatant was then ultracentrifuged at $100,000 \times \mathrm{g}$ for $30 \mathrm{~min}$. The microsomal pellet was resuspended in $60 \mathrm{~mL}$ of $0.6 \mathrm{M} \mathrm{KCl}, 0.3 \mathrm{M}$ sucrose, $20 \mathrm{mM}$ MOPS-Na (pH 7.4), and the protease inhibitor cocktail, and ultracentrifuged again. Finally, the pellet was resuspended in $12 \mathrm{~mL}$ of $0.3 \mathrm{M}$ sucrose, $20 \mathrm{mM}$ MOPS-Na ( $\mathrm{pH} 7.4$ ), and the protease inhibitor cocktail, followed by quick freezing in liquid nitrogen and storage at $-80^{\circ} \mathrm{C}$ until further use.

\section{Purification of RyR2}

RyR2 was purified using SBP-FKBP12.6 affinity chromatography (Cabra et al., 2016) with some modifications. The microsomes were solubilized with 2\% (w/v) CHAPS (Dojindo) and 1\% (w/v) soybean lecithin (Avanti Polar Lipids) in $1 \mathrm{M} \mathrm{NaCl}, 20 \mathrm{mM}$ MOPS (pH 7.4), 2 mM dithiothreitol, and the protease inhibitor cocktail for $30 \mathrm{~min}$ on ice. After centrifugation at $100,000 \times \mathrm{g}$ for $30 \mathrm{~min}$ at $4{ }^{\circ} \mathrm{C}$, 
the supernatant was diluted with four volumes of $20 \mathrm{mM}$ MOPS ( $\mathrm{pH} 7.4$ ), $2 \mathrm{mM}$ dithiothreitol, and the protease inhibitor cocktail, after which it was passed through a $0.45-\mu \mathrm{m}$ filter and loaded onto a preequilibrated 5-mL StrepTrap HP column (GE Healthcare, Chicago, IL) with bound SBP-FKBP12.6 fusion protein. The column was successively washed with 10 column volumes of (1) wash buffer (20 $\mathrm{mM}$ MOPS pH7.4, $2 \mathrm{mM}$ dithiothreitol, and $0.3 \mathrm{M}$ sucrose) containing $0.2 \mathrm{M} \mathrm{NaCl}$ and $0.25 \%(\mathrm{w} / \mathrm{v})$ CHAPS and (2) wash buffer containing $0.5 \mathrm{M} \mathrm{NaCl}$ and $0.015 \%$ (w/v) Tween-20 (Sigma-Aldrich). The SBP-FKBP12.6-RyR2 complex was eluted with the wash buffer supplemented with $2.5 \mathrm{mM}$ Ddesthiobiotin (Iba Lifesciences). After checking the purity by SDS-PAGE, the eluate was quickly frozen in liquid nitrogen and stored at $-80^{\circ} \mathrm{C}$ until further use.

\section{Negative staining}

The purified RyR2 sample was diluted with a buffer containing $0.2 \mathrm{M} \mathrm{NaCl}, 20 \mathrm{mM}$ MOPS-Na (pH 7.4), $2 \mathrm{mM}$ dithiothreitol, and $0.015 \%$ (w/v) Tween-20, and then applied to pre-hydrophilized carboncoated EM grids (400 mesh hexagonal copper grids, Stork Veco BV, Netherlands), negatively stained with $1.4 \%(\mathrm{w} / \mathrm{v})$ uranyl acetate solution, and observed at 40,000× magnification using a transmission electron microscope (H7500; Hitachi High-Technologies, Tokyo, Japan) operating at $80 \mathrm{kV}$. Micrographs were taken at 40,000 $\times$ using a 1,024 × 1,024 pixel CCD camera (Fast Scan-F114; TVIPS, Gauting, Germany).

\section{Cryo-EM sample preparation}

The purified RyR2 sample was buffer-exchanged and concentrated to $\sim 5 \mathrm{mg} / \mathrm{mL}$ using Amicon Ultra 100k (Millipore, Burlington, MA) with a buffer containing $0.5 \mathrm{M} \mathrm{NaCl}, 20 \mathrm{mM}$ MOPS-Na (pH 7.4), 2 $\mathrm{mM}$ dithiothreitol, and $0.015 \%\left(\mathrm{w} / \mathrm{v}\right.$ ) Tween-20. EGTA (final concentration of $1 \mathrm{mM}$ ) or $\mathrm{CaCl}_{2}$ (final concentration of $100 \mu \mathrm{M}$ ) was added to the concentrated protein samples to fix the channel to the closed or open state. The concentrated RyR2 was loaded onto a Quantifoil Cu/Rh grid (R1.2/1.3, 300 mesh) (Quantifoil), blotted using Vitrobot Mark III (FEI) with $4 \mathrm{~s}$ of blotting time and $100 \%$ humidity at $6{ }^{\circ} \mathrm{C}$, and then plunge-frozen in liquid ethane.

\section{Cryo-EM data collection}

Grids were screened for ice quality and cryo-EM data were acquired using a Titan Krios G3i cryo-EM (Thermo Fisher Scientific, Waltham, MA) running at $300 \mathrm{kV}$ and equipped with a Gatan Quantum-LS 
Energy Filter (slit width $25 \mathrm{eV}$ ) and a Gatan K3 Summit direct electron detector in the electron counting mode. The electron flux was set to $14 \mathrm{e}$-/pix/s at the detector. For the WT close state with EGTA and F4888A with EGTA, imaging was performed at a nominal magnification of $87,000 \times$, corresponding to a calibrated pixel size of $1.07 \AA /$ pix (University of Tokyo, Japan). Electron dose was set to $50 \mathrm{e}^{-} / \AA^{2}$ for the WT close state and $48 \mathrm{e} / / \AA^{2}$ for F4888A with EGTA. For other samples, imaging was performed at a nominal magnification of $105,000 \times$, corresponding to a calibrated pixel size of $0.83 \AA /$ pix. Electron

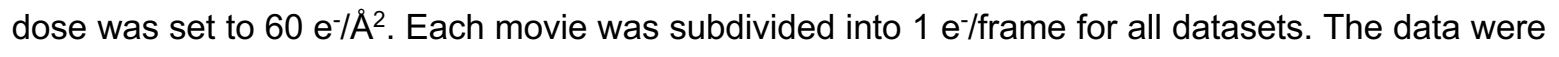
automatically acquired by the image shift method using SerialEM software (Mastronarde, 2005), with a defocus range of -0.8 to $-1.6 \mu \mathrm{m}$.

\section{Image processing}

Unless otherwise stated, the same procedure was used to process the data. The movie frames were motion corrected by RELION and CTF parameters and estimated by CTFFIND4 (Rohou and Grigorieff, 2015). All the following processes were performed using RELION (ver. 3.0 and 3.1) (Zivanov et al., 2018). To generate 2D templates for automatic particle picking, particles were picked from 100 randomly selected micrographs using template-free Laplacian-of-Gaussian picking, then subjected to multiple rounds of reference-free 2D classification. Good 2D classes were selected as templates and 2D template-based particle picking was performed.

Picked particles were extracted with $4 \times$ down-sampling and subjected to one round of 2D classification. Selected good particles from the 2D classification were submitted to 3D classification. After one round of $3 \mathrm{D}$ classification, good particles were selected, re-extracted with $1.5 \times$ downsampling, and subjected to 3D refinement. The resulting 3D map and particle set were then subjected to beam-tilt and per-particle defocus refinement, Bayesian polishing, and second round of per-particle defocus refinement followed by $3 \mathrm{D}$ refinement. To separate the particles based on open/close states, the no-align 3D classification was performed using a mask covering the TM region. Good classes were subjected to final $3 \mathrm{D}$ refinement and postprocessing. Resolution was determined according to the Fourier shell collation $(F S C)=0.143$ criterion.

For F4888A EGTA, two datasets were acquired, and particles were combined after Bayesian polishing. In each dataset, particles were selected from one round of 3D classification following two rounds of 2D classification. Detailed information is listed in Supplementary table 1 and described in figs. S2, S13, and S14. 


\section{Modelbuilding, refinement, and analysis}

Model building was performed using COOT (Emsley et al., 2010). RyR1 in the closed state (PDB accession code, 5TB0) was used as the initial reference model. The coordinates were rigid-body fitted in UCSF Chimera (Pettersen et al., 2004). After substitutions in mouse RyR2 sequence and manual building of the model, real space refinement was performed with PHENIX (Adams et al., 2010; Afonine et al., 2018) with secondary structure and geometry restrained. The residue sequences (110, 85-108, 861-864, 954-969, 1,015-1,026, 1,063-1,083, 1,275-1,283, 1,447-1,565, 1,851-1,890, 2,010-2,055, 2,362-2,378, 2,443-2,451, 2,659-2,711, 2,757-2,760, 2,785-2,834, 2,906-2,915, 2,943-2,960, 3,030-3,103, 3,130-3,135, 3,221-3,230, 3,435-3,476, 3,580-3,610, 3,649-3,658, $3,700-3,711,4,206-4,272,4,312-4,477,4,522-4,555$, and 4,963-4,966) were omitted, as the corresponding densities were not visible in all of the maps. All figures were prepared using PyMOL (The PyMOL Molecular Graphics System; http://www.pymol.org). Pore radii along the ion conducting pathway were calculated with HOLE (Smart et al., 1993). Buried surface areas were calculated with CNS (Brünger et al., 1998).

\section{$\left[{ }^{3} \mathrm{H}\right]$ Ryanodine binding}

$\left[{ }^{3} \mathrm{H}\right]$ Ryanodine binding assay was carried out as described previously (Fujii et al., 2017; Murayama et al., 2018a). Briefly, microsomes prepared from HEK293 cells expressing RyR2 were incubated for $1 \mathrm{~h}$ at $25^{\circ} \mathrm{C}$ with $5 \mathrm{nM}\left[{ }^{3} \mathrm{H}\right]$ ryanodine (PerkinElmer) in reaction media containing $0.17 \mathrm{M} \mathrm{NaCl}, 20 \mathrm{mM}$ MOPSO-Na ( $\mathrm{pH}$ 7.0), $2 \mathrm{mM}$ dithiothreitol, $1 \mathrm{mM} \mathrm{AMP}$, and $1 \mathrm{mM} \mathrm{MgCl}$. Free $\mathrm{Ca}^{2+}$ was adjusted with $10 \mathrm{mM}$ EGTA using WEBMAXC STANDARD (https://somapp.ucdmc.ucdavis.edu/pharmacology/bers/maxchelator/webmaxc/webmaxcS.htm). The $\left[{ }^{3} \mathrm{H}\right]$ ryanodine binding data $(B)$ were normalized to the maximum number of functional channels $\left(B_{\max }\right)$, which was separately determined by Scatchard plot analysis using various concentrations (3$20 \mathrm{nM}$ ) of $\left[{ }^{3} \mathrm{H}\right]$ ryanodine in a high-salt medium. The resultant $B / B_{\max }$ represents the averaged activity of each mutant.

\section{Single-cell $\mathrm{Ca}^{2+}$ imaging}

Single-cell $\mathrm{Ca}^{2+}$ imaging was performed using HEK293 cells expressing WT or mutant RyR2. In $\mathrm{Ca}^{2+}$ measurements with RyR2, the $\mathrm{Ca}^{2+}$ signals from the cytoplasm $\left(\left[\mathrm{Ca}^{2+}\right]_{\mathrm{cyt}}\right)$ and ER lumen $\left(\left[\mathrm{Ca}^{2+}\right]_{\mathrm{ER}}\right)$ were monitored using G-GECO1.1 (a gift from Robert Campbell from University of Alberta; Addgene 
plasmid \#32445) (Zhao et al., 2011). and R-CEPIA1er (a gift from Masamitsu lino, Nihon University, Tokyo, Japan; Addgene plasmid \#58216) (Suzuki et al., 2014), respectively. Cells were transfected with cDNAs for these $\mathrm{Ca}^{2+}$ indicators $26-30 \mathrm{~h}$ before measurement and at the same time doxycycline $(2 \mu \mathrm{g} / \mathrm{mL})$ was added to the culture medium to induce RyR2 expression. Experiments were carried out in HEPES-buffered Krebs solution ( $140 \mathrm{mM} \mathrm{NaCl}, 5 \mathrm{mM} \mathrm{KCl}, 2 \mathrm{mM} \mathrm{CaCl}, 1 \mathrm{mM} \mathrm{MgCl}$, $11 \mathrm{mM}$ glucose, and $5 \mathrm{mM}$ HEPES at $\mathrm{pH} 7.4$ ). At the end of each experiment, $F_{\min }$ and $F_{\max }$ were obtained with 0Ca Krebs solution containing $20 \mu \mathrm{M}$ ionomycin, $5 \mathrm{mM}$ BAPTA, and $20 \mu \mathrm{M}$ cyclopiazonic acid and $20 \mathrm{Ca}$ Krebs solution containing $20 \mu \mathrm{M}$ ionomycin and $20 \mathrm{mM} \mathrm{CaCl}_{2}$, respectively (Uehara et al., 2017). The fluorescence signal $\left(F-F_{\min }\right)$ was normalized to the maximal fluorescence intensity $\left(F_{\max }-F_{\min }\right)$. Measurements were carried out at $26^{\circ} \mathrm{C}$. 


\section{Titles for multimedia files}

\section{Video S1.}

The architecture of RyR2 and details of its opening.

\section{Video S2.}

Rotation of C-central/U-motif/U-motif/S6 $6_{\text {cyto }} / \mathrm{CTD}$ complex upon $\mathrm{Ca}^{2+}$ binding.

\section{Video S3.}

Details of signal transmission from the cytoplasm into the TM region via the U-motif/S2-S3 domain interaction.

Video S4.

Conformation changes in the TM region upon $\mathrm{Ca}^{2+}$ binding.

\section{Video S5.}

Details of the U-motif/S6yto/CTD interaction.

\section{Video S6.}

Structure comparison of the F4888A mutant with the WT in the closed state. 

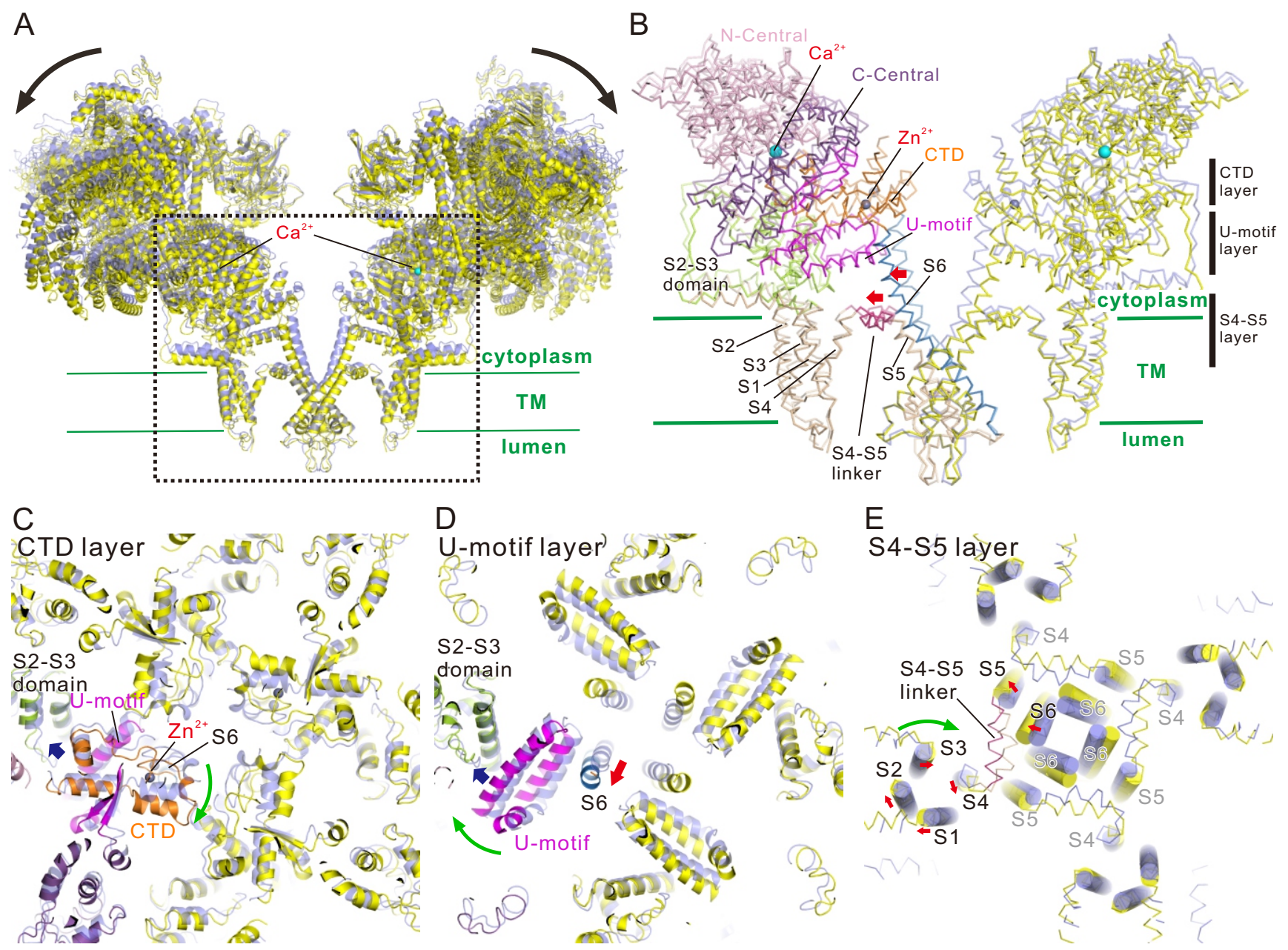

U-motif layer

E
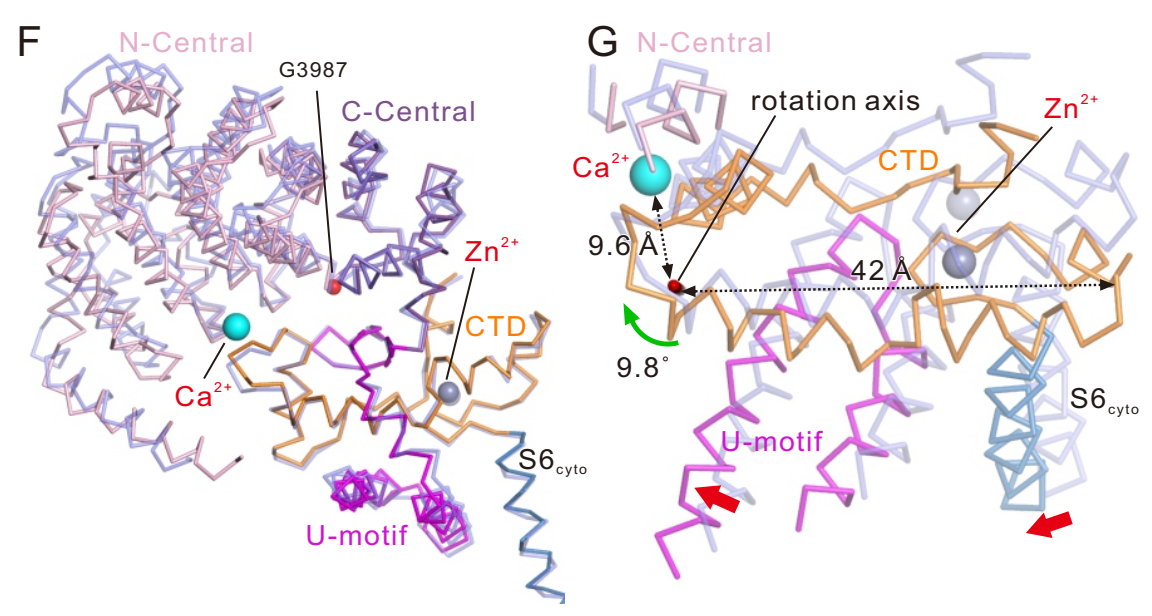

$\mathrm{H}$

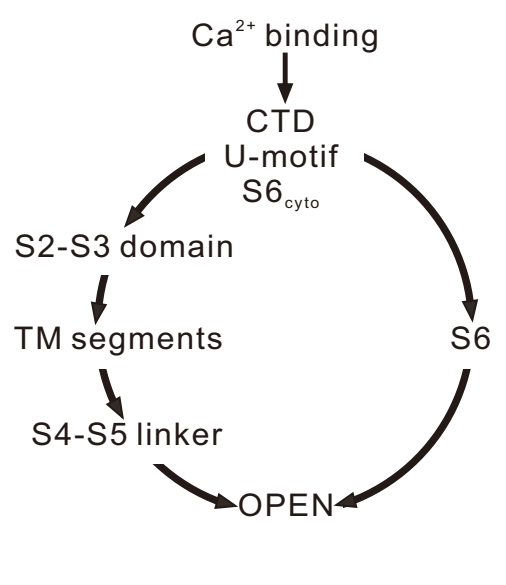

Figure 1 

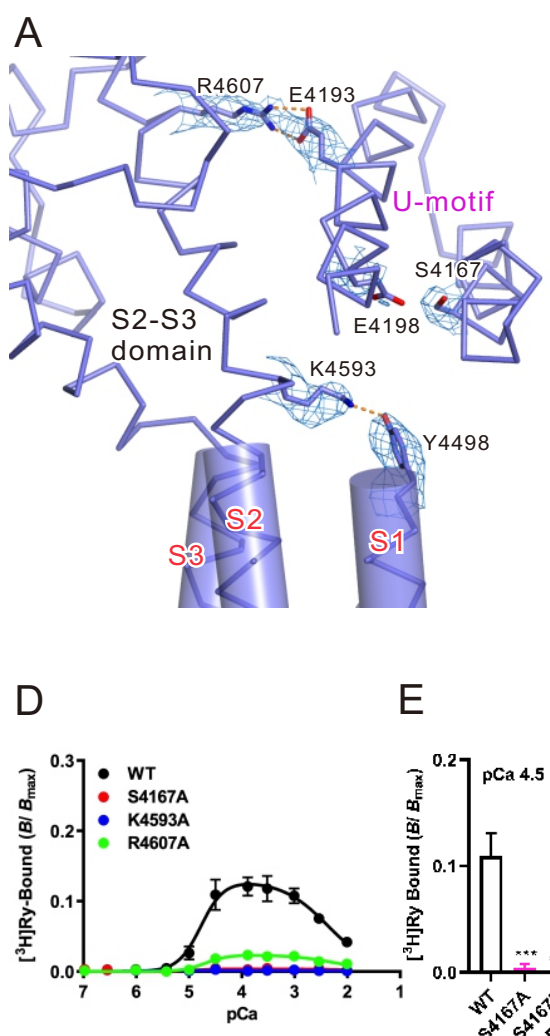

$E$

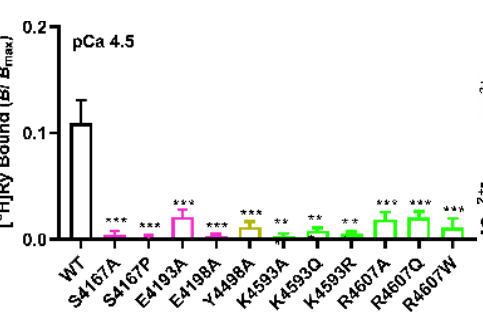

$\mathrm{F}$
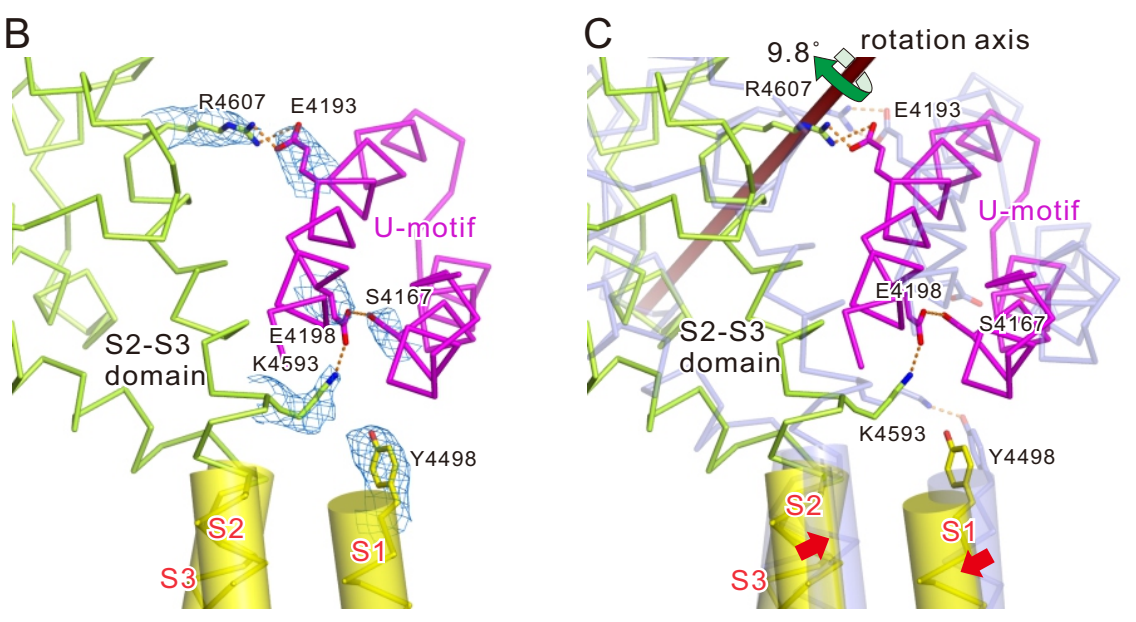

$\mathrm{H}$

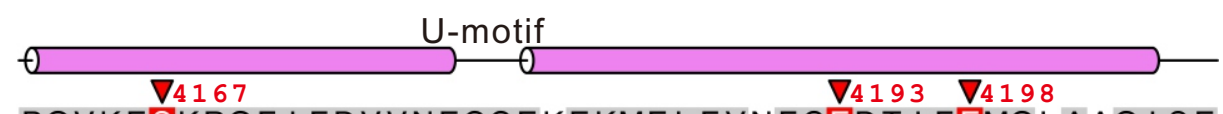

mRYR2 4162 PQVKESKRQF I FDVVNEGGEKEKMELFVNFCEDT I FEMQLAAQ I SE 4207 hRYR2 4163 PQVKESKRQF I FDVVNEGGEKEKMEL FVNFCEDT I FEMQLAAQ I SE 4208 PRYR2 4183 PQVKESKRQF I FDVVNEGGEKEKMELFVNFCEDT I FEMQLAAQ I SE 4228 rRYR1 4208 PQVKESKRQF I FDVVNEGGEAEKME LFVSFCEDT I FEMQ I AAQ I SE 4251 hRYR1 4207 PQVKESKRQF I FDVVNEGGEAEKMELFVSFCEDT I FEMQ I AAQ I SE 4252 hRYR3 4059 PQVKESKRQF I FDVVNEGGEQEKMEL FVNFCEDT I FEMQLASQ I SE 4104

S1

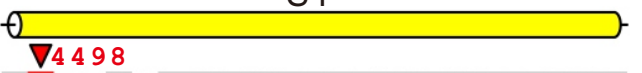

4497 FYNMRML A L FVAFA I NF I L LFYKV 4520 4499 F YNMRML ALFVAFA I NF I L LFYKV 4522 4519 FYNMRMLALFVAFA I NF I L LFYKV 4542 4559 FYTLRFLALFVAFA I NF I LLFYKV 4582 4560 FYT LRFLALFVAFA I NF I LLFYKV4583 4410 FYNLRFLALFVAFA INFI LLFYKV 4433

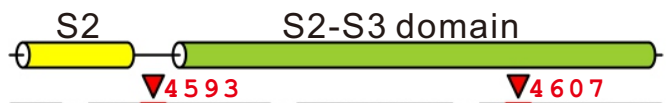

4588 GYYCLKVPLV I FKREKEVARKLEFD 4612 4589 GYYCLKVPLV I FKREKEVARKLEFD 4613 4609 GYYCLKVPLV I FKREKEVARKLEFD 4633 4660 GYNCLKVPLV I FKREKELARKLEFD 4684 4661 GYNCLKVPLV I FKREKELARKLEFD 4685 4494 GYYCLKVPL VVFKREKE I ARKLEFD 4518 
bioRxiv preprint doi: https://doi.org/10.1101/2020.11.30.401026; this version posted February 12, 2021. The copyright holder for this preprint (which was not certified by peer review) is the author/funder. All rights reserved. No reuse allowed without permission.

A

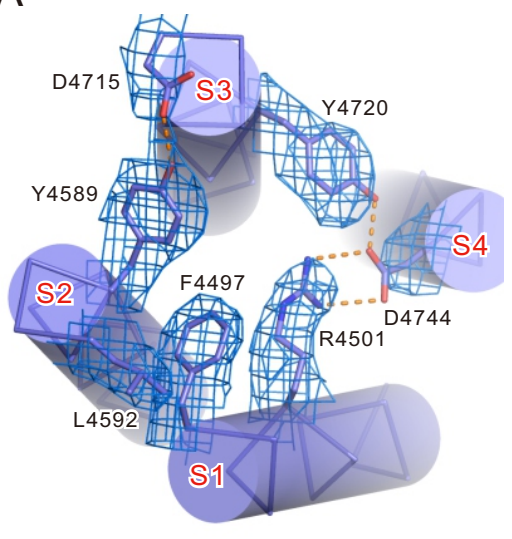

D

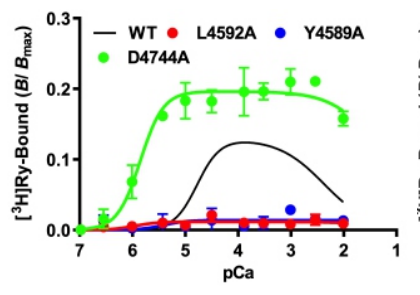

$\mathrm{H}$

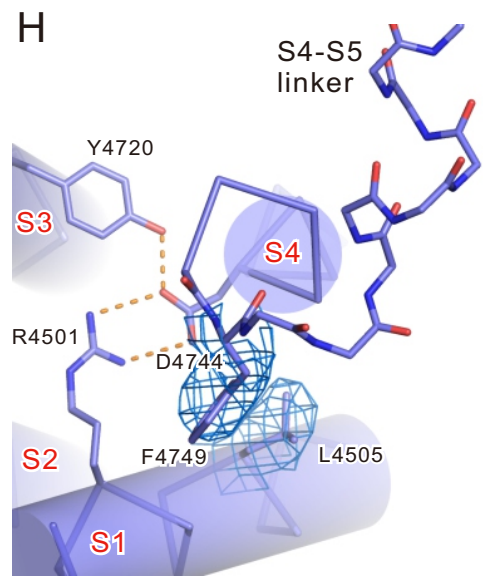

K

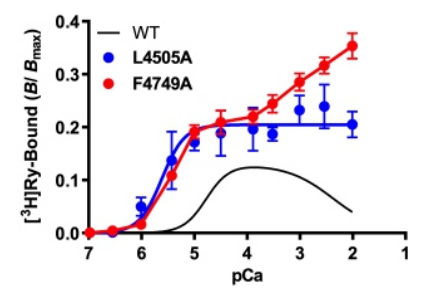

。

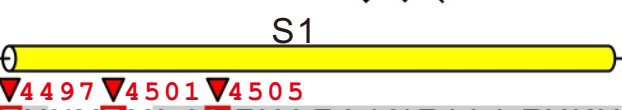

$B$

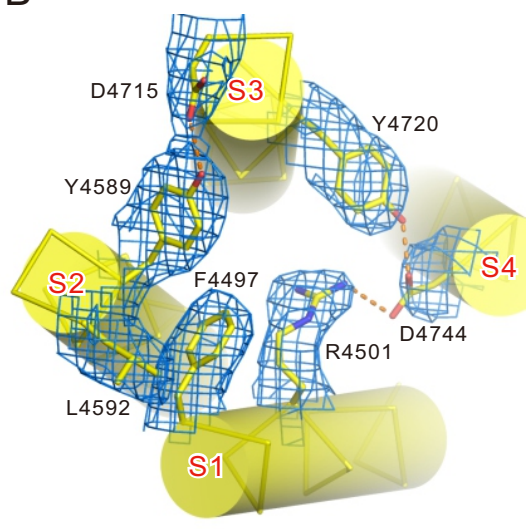

F

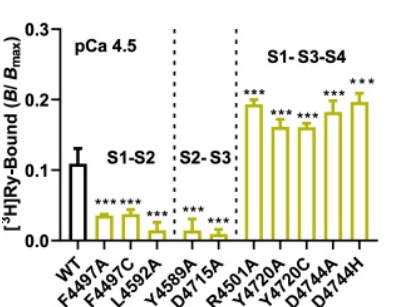

E
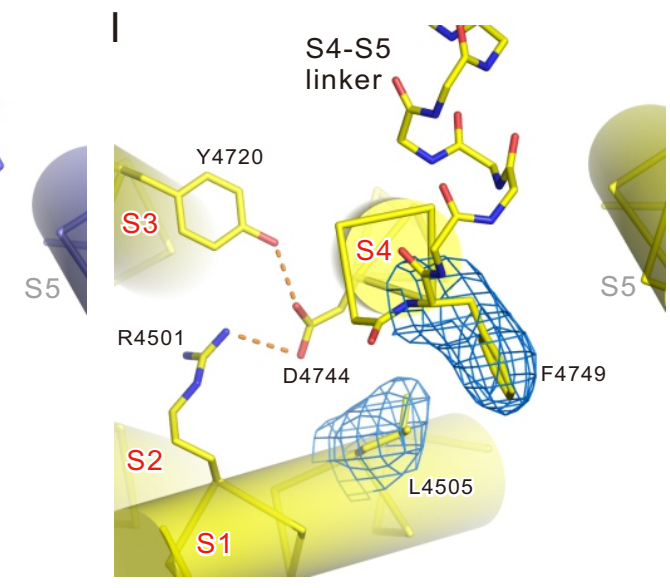

L

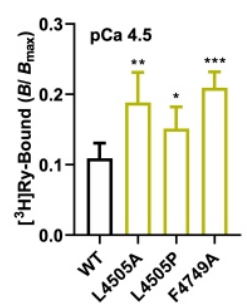

M

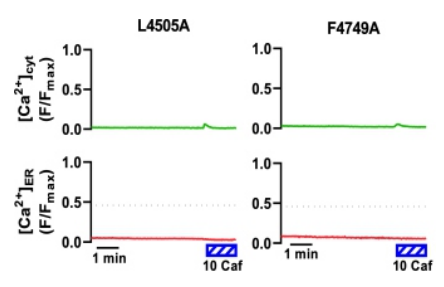

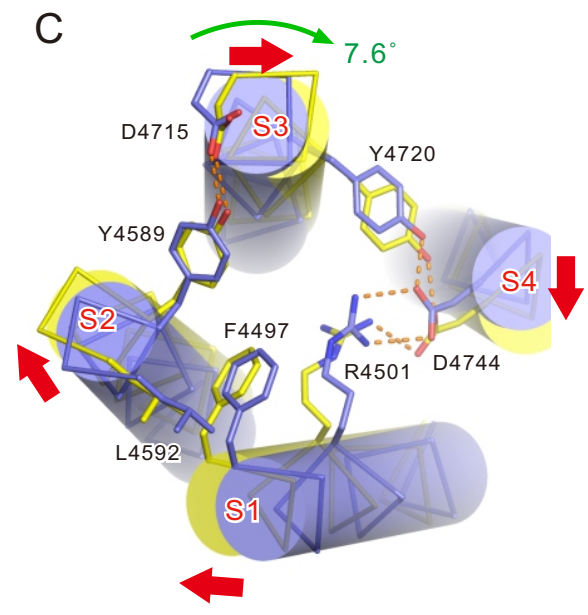

G
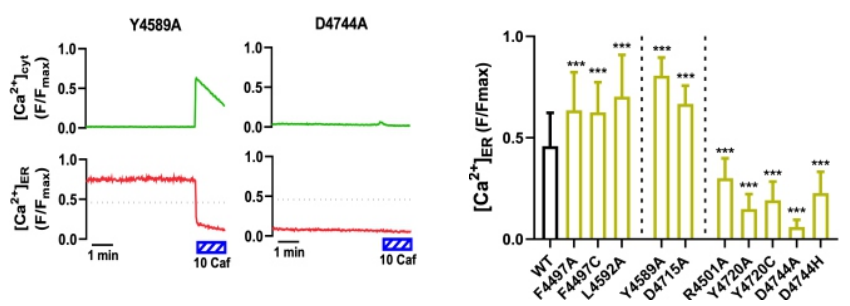

J

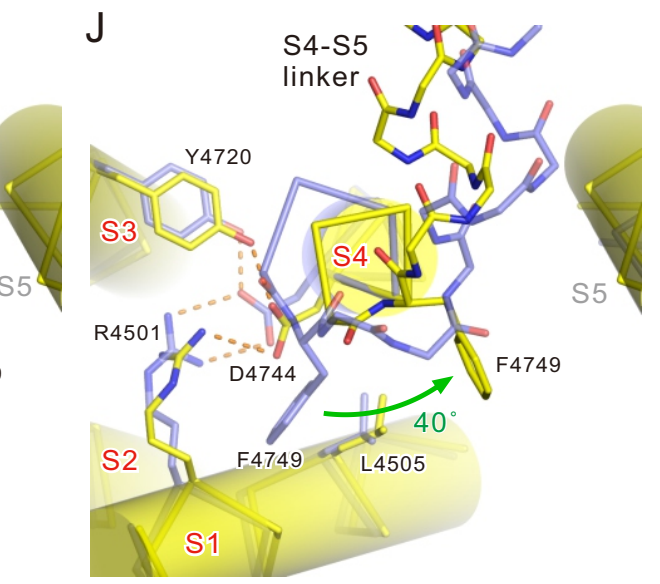

N

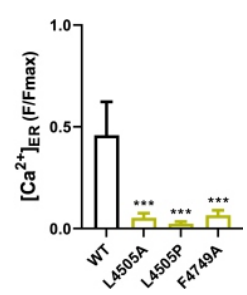

S2

mRYR2 4497 FYNMRML ALFVAFA INF I L LFYKV45204568EPTLRILA I LHTI I SFFC I IGYYCL 4592 hRYR2 4499 FYNMRMLALFVAFA I NF I LLFYKV45224569 EPTLR I LA I LHTV I SFFC I I GYYCL4593 PRYR2 4519 FYNMRMLALFVAFA I NF I LLFYKV45424589EPTLR I LA I LHTV ISFFC I I GYYCL 4613 rRYR1 4559 FYT LRFLALFVAFA I NF I LLFYKV45824640EPALWCLSLLHTLVAFLC I I GYNCL 4664 hRYR1 4560 FYTLRFLALFVAFA INF I LLFYKV 45834641 EPALRCLSLLHTLVAFLCI I GYNCL 4665 hRYR3 4410 FYNLRFLALFVAFA I NF I LLFYKV44334474APTLRALA I I HTI I SLVCVVGYYCL 4498

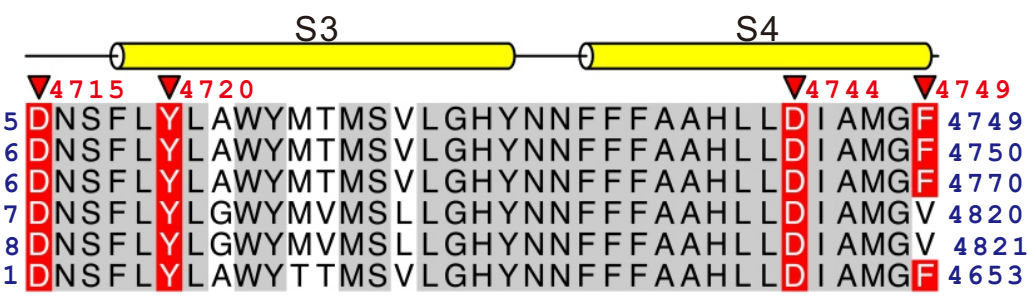

Figure 3 
A

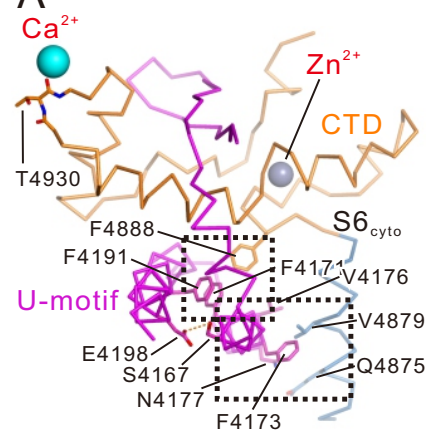

E

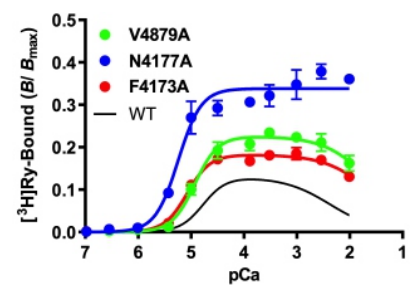

I

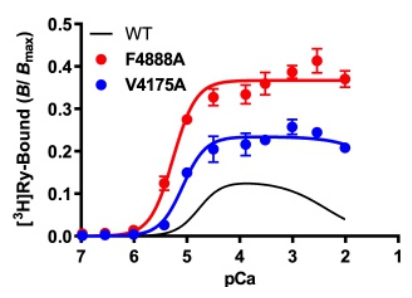

B

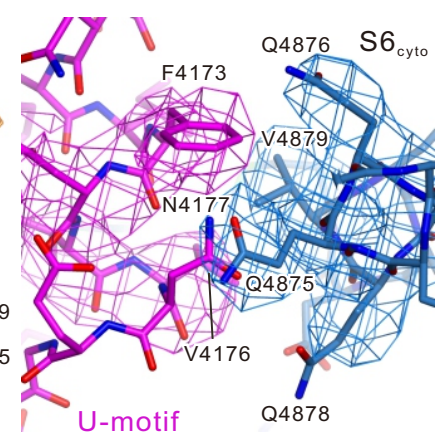

$\mathrm{F}$

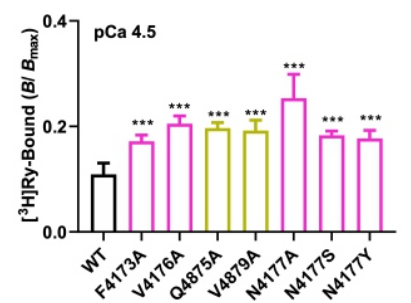

$\mathrm{J}$

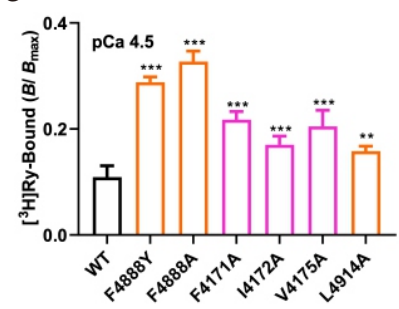

C

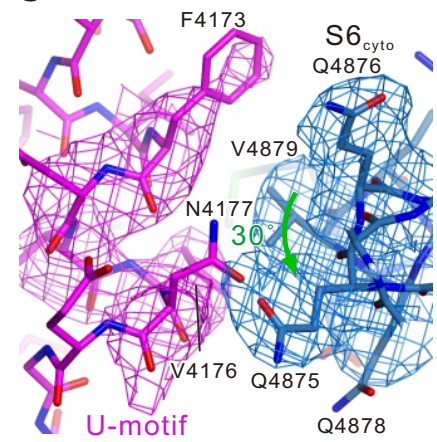

G

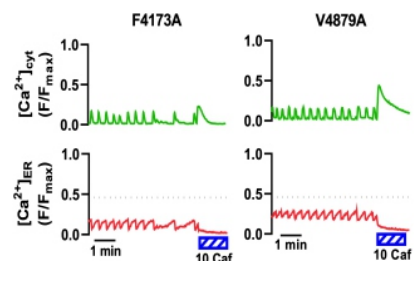

$\mathrm{K}$

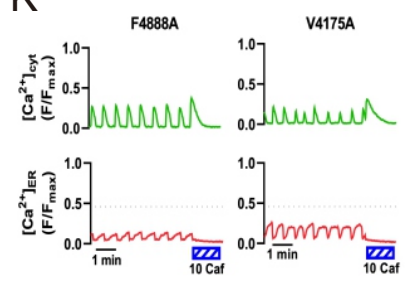

D

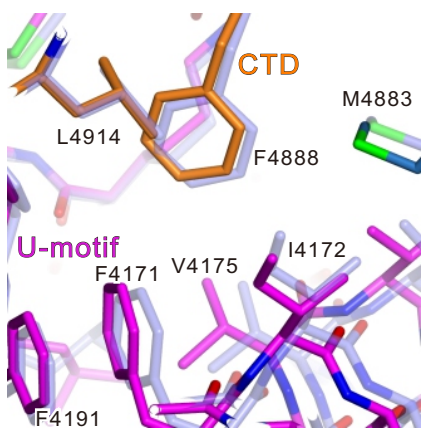

$\mathrm{H}$

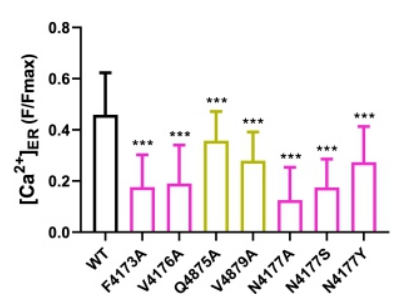

L

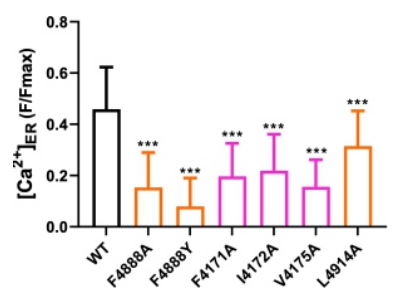

M

U-motif

$\begin{array}{ll}4171 \nabla \nabla \nabla & \nabla \nabla \nabla 4177\end{array}$

mRYR2 4162 PQVKESKRQF I FDVVNEGGEKEKME LFVNFCEDT I FEMQLAAQI SE 4207

hRYR2 4163 PQVKESKRQF I FDVVNEGGEKEKME LFVNFCEDT I FEMQLAAQ I SE 4208

PRYR2 4183 PQVKESKRQF I FDVVNEGGEKEKME LFVNFCEDT I FEMQLAAQ I SE 4228

IRYR 14208 PQVKESKRQF I FDVVNEGGEAEKMELFVSFCEDT I FEMQ I AAQ I SE 4251

hRYR 14207 PQVKESKRQF I FDVVNEGGEAEKMELFVSFCEDT I FEMQ I AAQ I SE 4252

hRYR3 4059 PQVKESKRQF I FDVVNEGGEQEKMELFVNFCEDT IFEMQLASQI SE 4104

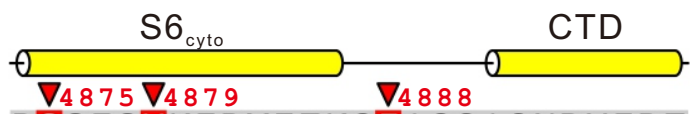

4874 DQQEQVKEDMETKCF I CG I GNDYFDT 4899

4875 DQQEQVKEDMETKCF I CG I GNDYFDT 4900

4895 DQQEQVKEDMETKCF I CG I GNDYFDT 4920

4945 DQQEQVKEDMETKCF I CG I GNDYFDT 4970

4946 DQQEQVKEDMETKCF I CG I GNDYFDT 4971

4778 DQQEQVKEDMETKCFI CG I GNDYFDT 4803 
A

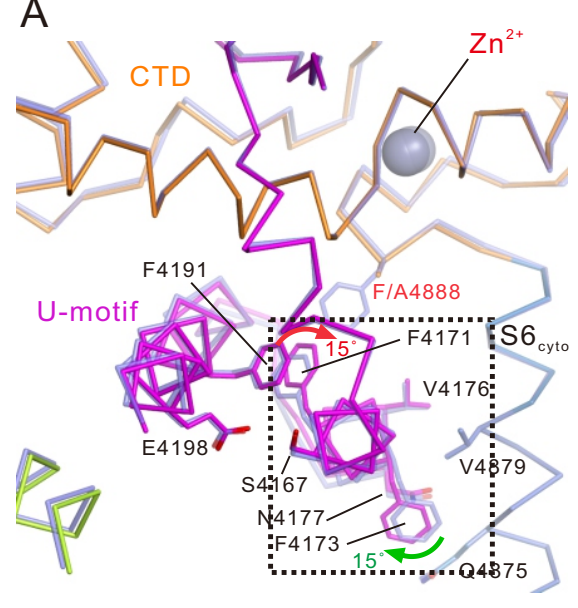

B

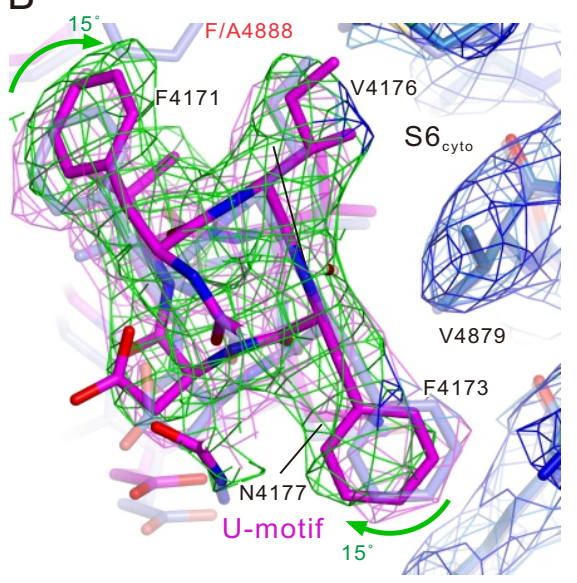

E
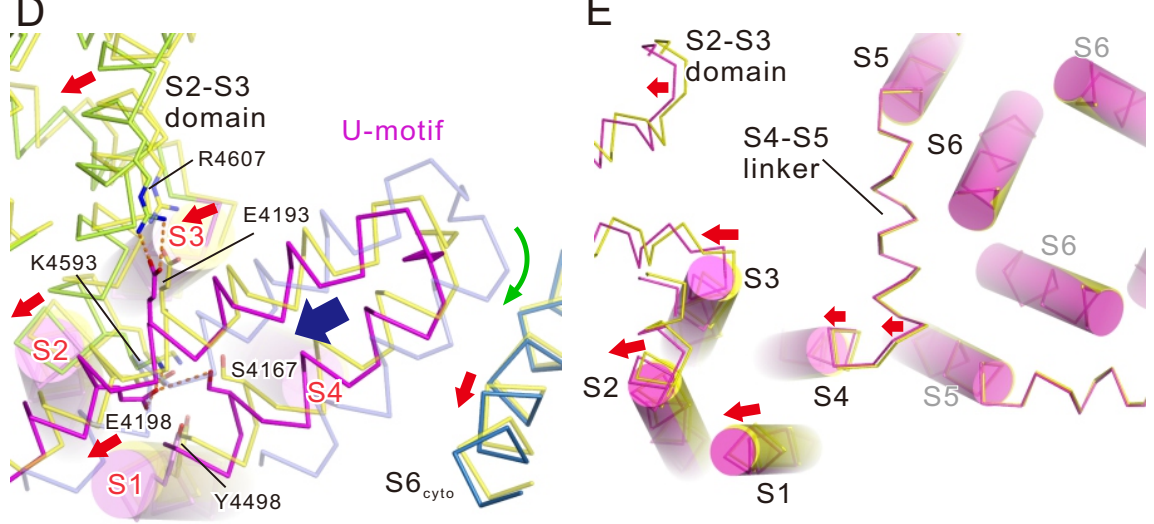

C

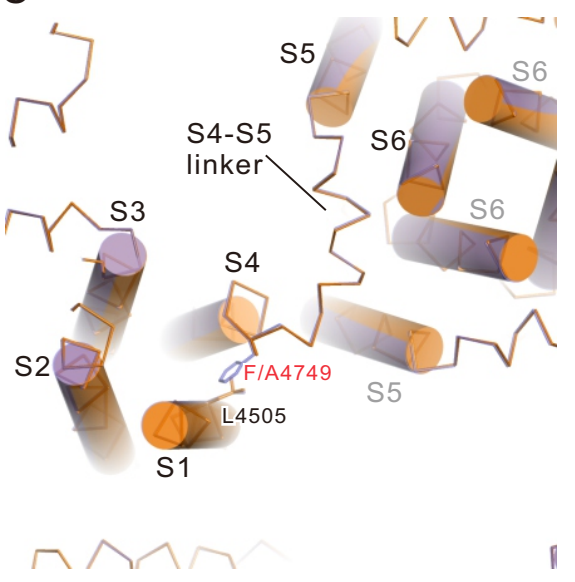

$\mathrm{F}$

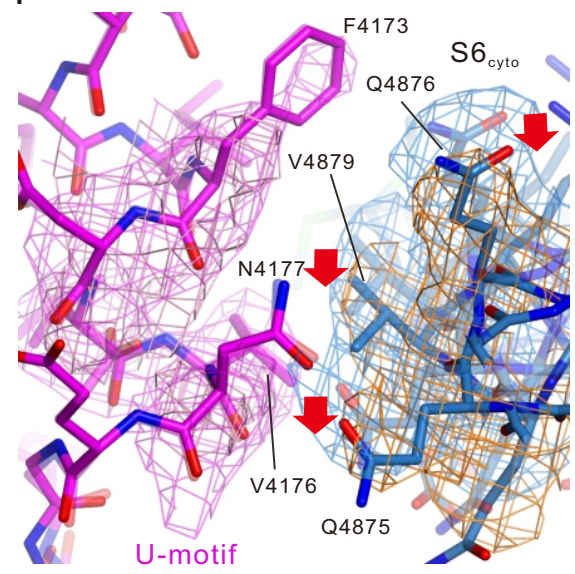

G

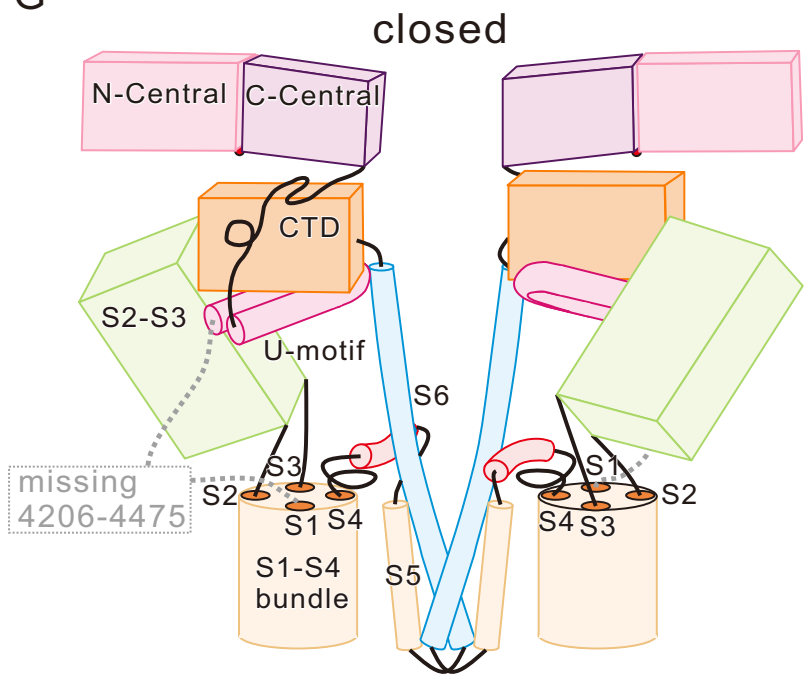

$\mathrm{H}$

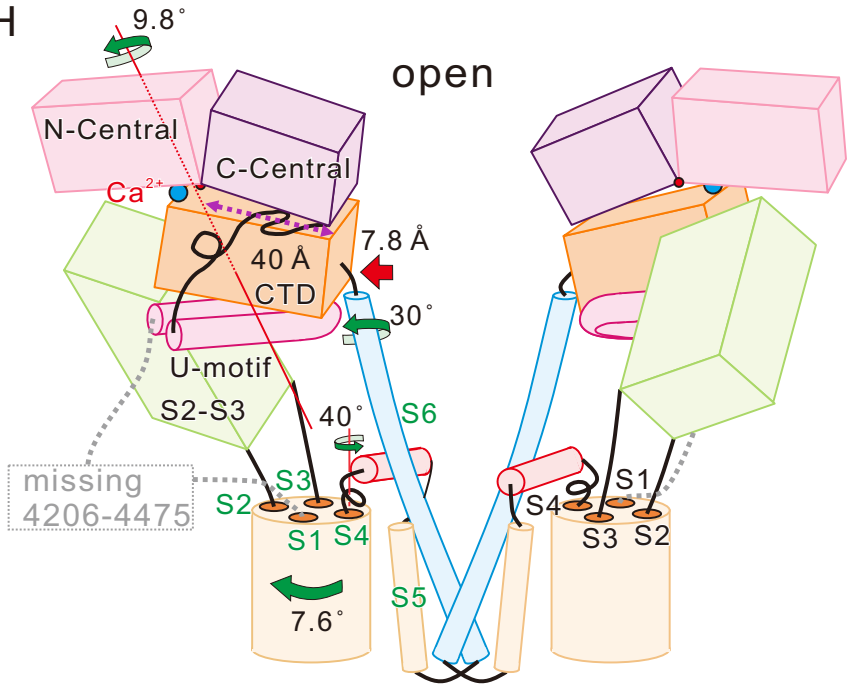



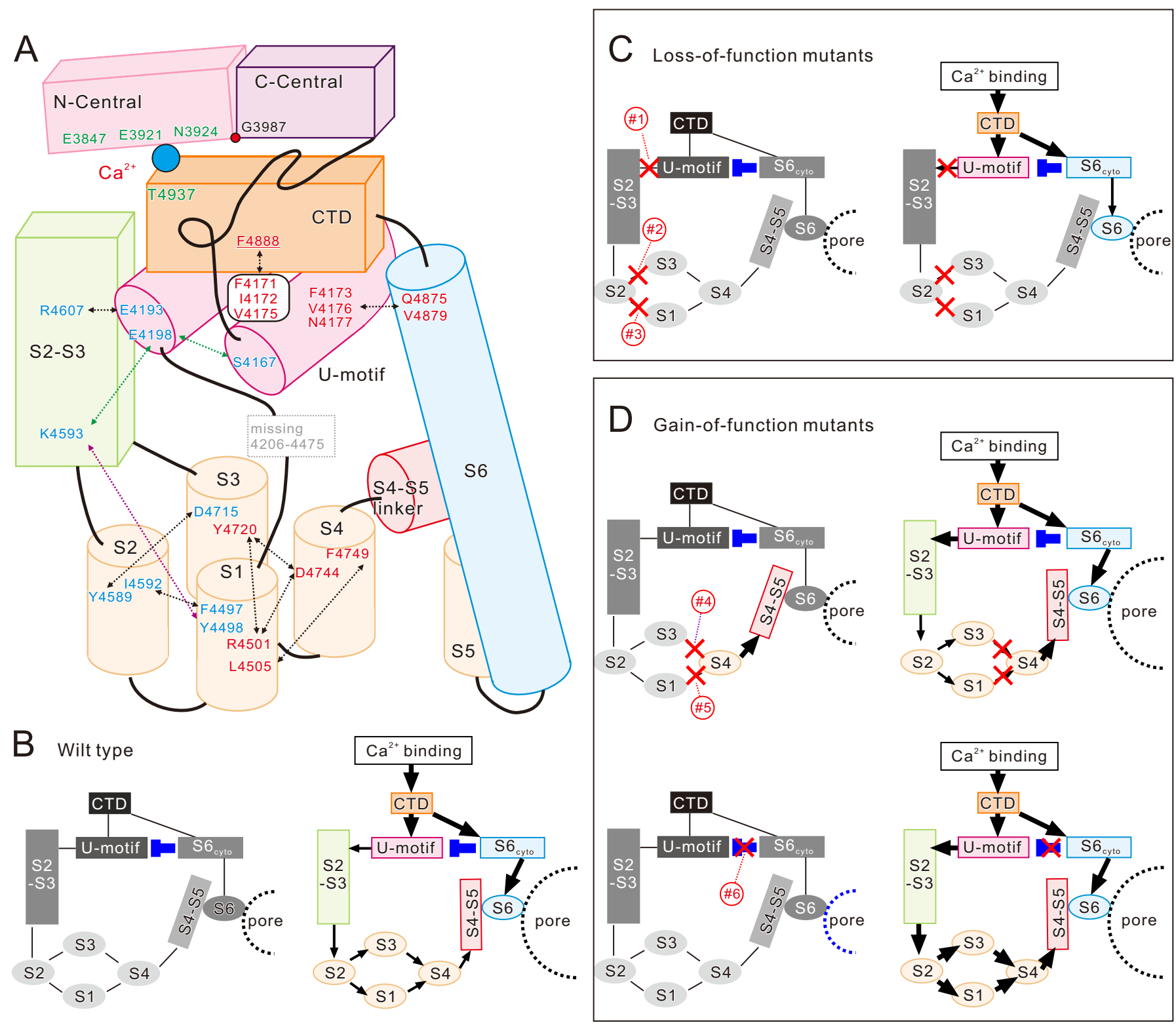